\title{
Tensorization of the strong data processing inequality for quantum chi-square divergences
}

\author{
Yu Cao ${ }^{1}$ and Jianfeng $\mathrm{Lu}^{1,2}$ \\ ${ }^{1}$ Department of Mathematics, Duke University, Box 90320, Durham NC 27708, USA \\ ${ }^{2}$ Department of Physics and Department of Chemistry, Duke University, Box 90320, Durham NC 27708, USA
}

It is well-known that any quantum channel $\mathcal{E}$ satisfies the data processing inequality (DPI), with respect to various divergences, e.g., quantum $\chi_{\kappa}^{2}$ divergences and quantum relative entropy. More specifically, the data processing inequality states that the divergence between two arbitrary quantum states $\rho$ and $\sigma$ does not increase under the action of any quantum channel $\mathcal{E}$. For a fixed channel $\mathcal{E}$ and a state $\sigma$, the divergence between output states $\mathcal{E}(\rho)$ and $\mathcal{E}(\sigma)$ might be strictly smaller than the divergence between input states $\rho$ and $\sigma$, which is characterized by the strong data processing inequality (SDPI). Among various input states $\rho$, the largest value of the rate of contraction is known as the SDPI constant. An important and widely studied property for classical channels is that SDPI constants tensorize. In this paper, we extend the tensorization property to the quantum regime: we establish the tensorization of SDPIs for the quantum $\chi_{\kappa_{1 / 2}}^{2}$ divergence for arbitrary quantum channels and also for a family of $\chi_{\kappa}^{2}$ divergences (with $\kappa \geq \kappa_{1 / 2}$ ) for arbitrary quantum-classical channels.

\section{Introduction}

In information theory, the data processing inequality (DPI) has been an important property for divergence measures to possess operational meaning. For instance, DPI has been proved for quantum $\chi_{\kappa}^{2}$ divergences (see e.g., [12, Thm. II.14] or [21, Thm. 4]), among other divergences. More explicitly, for any quantum channel $\mathcal{E}$ and for all quantum states $\rho, \sigma \in \mathfrak{D}_{n}$, we have

$$
\chi_{\kappa}^{2}(\mathcal{E}(\rho) \| \mathcal{E}(\sigma)) \leq \chi_{\kappa}^{2}(\rho \| \sigma) .
$$

In the above, $\kappa$ is a real-valued positive function (see (7) below); the definition of $\chi_{\kappa}^{2}$ divergences will be postponed to $\S 2.1$, as it involves some technicalities.

Compared with the DPI, the strong data processing inequality (SDPI) quantitatively and more precisely characterizes the extent that quantum states contract under the channel $\mathcal{E}$ [1, 16-18]. Given any $(\mathcal{E}, \sigma)$-pair where $\mathcal{E}$ is any quantum channel and $\sigma \in \mathfrak{D}_{n}^{+}$is any full-rank quantum state $\left(\mathfrak{D}_{n}^{+}\right.$is the space of strictly positive density matrices on a $n$-dimensional Hilbert space), if there is a constant $\eta_{\chi_{\kappa}^{2}}(\mathcal{E}, \sigma) \in[0,1)$ such that

$$
\chi_{\kappa}^{2}(\mathcal{E}(\rho) \| \mathcal{E}(\sigma)) \leq \eta_{\chi_{\kappa}^{2}}(\mathcal{E}, \sigma) \chi_{\kappa}^{2}(\rho \| \sigma), \forall \rho \in \mathfrak{D}_{n},
$$

then the quantum channel $\mathcal{E}$ is said to satisfy the strong data processing inequality (SDPI) for the quantum $\chi_{\kappa}^{2}$ divergence and the smallest constant $\eta_{\chi_{\kappa}^{2}}(\mathcal{E}, \sigma)$ such that $(2)$ holds is called the SDPI constant. Evidently,

$$
\eta_{\chi_{\kappa}^{2}}(\mathcal{E}, \sigma)=\sup _{\rho \in \mathfrak{D}_{n}: \rho \neq \sigma} \frac{\chi_{\kappa}^{2}(\mathcal{E}(\rho) \| \mathcal{E}(\sigma))}{\chi_{\kappa}^{2}(\rho \| \sigma)} .
$$

Many applications of SDPIs can be found in e.g., [17, Sec. 2.3] and [18, Sec. V].

Yu Cao: yucao@math.duke.edu
Jianfeng Lu: jianfeng@math.duke.edu 
It is common in quantum information theory to consider high-dimensional quantum channels, formed by the tensor product of low-dimensional quantum channels. Except for very special cases, in general, obtaining SDPI constants for high-dimensional quantum channels can be rather challenging, even numerically. It is desirable if one could reduce the problem of calculating the SDPI constant for a (global) high-dimensional quantum channel, to calculating the SDPI constants of low-dimensional quantum channels. For a specific divergence (e.g., quantum $\chi_{\kappa}^{2}$ divergence in this work), if the SDPI constant for the high-dimensional channel is the maximum value of SDPI constants for these low-dimensional channels, we say that the SDPI constant for this divergence satisfies the tensorization property.

Our main result in this work is that the SDPI constant for $\chi_{\kappa}^{2}$ tensorizes, summarized in the following theorem.

Theorem 1. Consider $N$ finite-dimensional quantum systems whose Hilbert spaces are $\mathcal{H}_{j}$ with dimension $n_{j}(1 \leq j \leq N)$ and consider any density matrix $\sigma_{j} \in \mathfrak{D}_{n_{j}}^{+}$and any quantum channel $\mathcal{E}_{j}$ acting on $\mathcal{H}_{j}$, such that for all $1 \leq j \leq N, \mathcal{E}_{j}\left(\sigma_{j}\right) \in \mathfrak{D}_{n_{j}}^{+}$. If either of the followings holds

(i) $\kappa=\kappa_{1 / 2}$;

(ii) $\kappa \geq \kappa_{1 / 2}$ and $\mathcal{E}_{j}$ are quantum-classical (QC) channels;

then we have the tensorization of the SDPI constant for the quantum $\chi_{\kappa}^{2}$ divergence, i.e.,

$$
\eta_{\chi_{\kappa}^{2}}\left(\mathcal{E}_{1} \otimes \mathcal{E}_{2} \otimes \cdots \otimes \mathcal{E}_{N}, \sigma_{1} \otimes \sigma_{2} \otimes \cdots \otimes \sigma_{N}\right)=\max _{1 \leq j \leq N} \eta_{\chi_{\kappa}^{2}}\left(\mathcal{E}_{j}, \sigma_{j}\right)
$$

Remark. (i) The function $\kappa_{1 / 2}(x):=x^{-1 / 2}$ is a special example of weight functions. There are some properties that only the quantum $\chi_{\kappa_{1 / 2}}^{2}$ divergence possesses (see e.g., Lemma 7 (ii)); in addition, $\chi_{\kappa_{1 / 2}}^{2}$ is tightly connected to the sandwiched Rényi divergence of order 2 [13].

There is a whole family of $\kappa_{\alpha}$ parameterized by $\alpha \in[0,1]$, satisfying the condition $\kappa_{\alpha} \geq \kappa_{1 / 2}$; see Example 3 for details; in $\S 2.2$, we also present other examples of $\kappa(x)$ such that $\kappa \geq \kappa_{1 / 2}$. The notion of QC channel will be recalled in $\S 2.6$.

(ii) These assumptions only provide sufficient conditions for the tensorization of SDPIs to hold, and it is an interesting open question to further investigate weaker conditions. In addition, it is also an interesting open question whether the tensorization of SDPIs holds for (quasi) relative entropies and the geodesic distances $[8,12]$. We shall leave these questions to future research.

The tensorization property in the classical regime has been well studied and widely used; see e.g., [1, 18, 22]. For SDPI constants, the tensorization property was proved in [18, Thm. III.9] for any $\Phi$-divergence, denoted by $D_{\Phi}(\nu \| \mu):=\mathbb{E}_{\mu}\left[\Phi\left(\frac{\mathrm{d} \nu}{\mathrm{d} \mu}\right)\right]-\Phi(1)$, provided that the associated $\Phi$-entropy is sub-additive and homogeneous. As a remark, the $\Phi$-divergence includes the relative entropy (with $\Phi(x)=x \log (x)$ ) and the classical $\chi^{2}$ divergence (with $\Phi(x)=(x-1)^{2}$ ) as special instances. The tensorization of SDPI constants associated with the classical relative entropy has been applied to study the lower bounds of Bayes risk [24].

Establishing tensorization in the quantum regime seems to be more challenging and our understanding is much limited. Recently, the tensorization technique has been developed for the quantum hypercontractivity of qubit system [11], reversed hypercontractivity [3, 7], 2-log-Sobolev constant [3, 10], as well as the quantum maximal correlation [2]. For the tensorization of the quantum (reversed) hypercontractivity and log-Sobolev constants, all existing works, as far as we know, focus exclusively on reversible (or even more special) quantum Markov semigroups (i.e., Lindblad equations).

We would like to briefly mention and highlight the proof techniques used for Theorem 1 . The first main ingredient is to formulate the SDPI constant as the second largest eigenvalue of a certain operator (see Lemma 10); similar results have been obtained in e.g., [5, 8, 12, 18, 20]. This result immediately leads into the proof of the case (i). The second main ingredient is to bound $\eta_{\chi_{\kappa_{1 / 2}}^{2}}(\mathcal{E}, \sigma)$ above by $\sqrt{\eta_{\chi_{\kappa}^{2}}(\mathcal{E}, \sigma)}$ (see Lemma 12), whose proof uses Petz recovery map [14] as the bridge. This relation together with special properties of $\eta_{\chi_{\kappa_{1 / 2}}^{2}}(\mathcal{E}, \sigma)$ leads into the proof of case (ii). 


\section{Related techniques to quantify the loss of information}

Apart from the DPI and the SDPI, there are other concepts used to characterize the contraction of quantum states under the action of noisy channels. For instance, one widely studied quantity is the contraction coefficient

$$
\eta_{\chi_{\kappa}^{2}}(\mathcal{E}):=\sup _{\sigma \in \mathfrak{D}_{n}^{+}} \eta_{\chi_{\kappa}^{2}}(\mathcal{E}, \sigma)
$$

The contraction coefficient is very similar to the SDPI constant. However, compared with the SDPI constant, the contraction coefficient for various divergence measures has been much more extensively studied in the literature, see e.g., [8, 12, 15, 20, 21] for the quantum case, and see e.g., [6] for the classical case. The bijection maps that preserve the quantum $\chi_{\kappa_{\alpha}}^{2}$ divergence (see Example 3 about the family $\kappa_{\alpha}$ ) have been characterized in [4], which complements the study of the contraction of quantum states. There are other tools based on the functional perspective, including quantum (reverse) hypercontractivity and related quantum functional inequalities [3, 7, 10, 11, 19].

\section{Contribution}

We summarize new results obtained in this work, as follows:

(i) Our main result is Theorem 1, which establishes the tensorization of SDPI constants, under certain assumptions: for the quantum $\chi_{\kappa_{1 / 2}}^{2}$ divergence, the tensorization of SDPI constants holds for general quantum channels; for the quantum $\chi_{\kappa}^{2}$ divergence with $\kappa \geq \kappa_{1 / 2}$, the tensorization holds for any quantum-classical channel.

(ii) Along the analysis of the SDPI, we also establish a connection between the SDPI constant associated with $\kappa_{1 / 2}$ and a variant of quantum maximal correlations; see Theorem 18 for details.

(iii) To use the tensorization property, we need to understand the SDPI constants for local channels, i.e., we need to compute $\eta_{\chi_{\kappa}^{2}}\left(\mathcal{E}_{j}, \sigma_{j}\right)$ for $1 \leq j \leq N$. Motivated by this, we study the SDPI constants for special qubit channels in $\S 5$. We notice that there is a particular QC channel $\mathcal{E}$ associated with a fixed $\sigma \in \mathfrak{D}_{2}^{+}$such that the largest value of $\eta_{\chi_{\kappa}^{2}}(\mathcal{E}, \sigma) \approx 1$ for $\kappa=\kappa_{\min }$, while $\eta_{\chi_{\kappa}^{2}}(\mathcal{E}, \sigma) \approx 0$ for $\kappa=\kappa_{\max }$ (however, $\sigma$ is close to a singular matrix); see $\S 5.1$ for details. This extreme example shows the high dependence of SDPI constants on the choice of $\kappa$, which magnifies the difference between the quantum SDPI constant and its classical analog, because there is only one SDPI constant for the classical $\chi^{2}$ divergence.

This paper is organized as follows. In $\S 2$, we provide some preliminary results, in particular, we recall the eigenvalue formalism of the SDPI constant. In $\S 3$, we prove Theorem 1 and in $\S 4$, we study the connection between the SDPI constant and the quantum maximal correlation. In $\S 5$, we consider SDPI constants for qubit channels and study the dependence of $\eta_{\chi_{\kappa}^{2}}(\mathcal{E}, \sigma)$ on $\sigma$ and $\kappa$. $\S 6$ concludes the paper with some additional remarks.

\section{Preliminaries}

This section contains preliminary results that we will use to prove the tensorization of the strong data processing inequality, Theorem 1. In particular, we will present two variational formulations of SDPI constants, and discuss the relation between various SDPI constants.

Notations. We shall consider finite dimensional systems only, i.e., the Hilbert space $\mathcal{H} \cong \mathbb{C}^{n}$. Let $\mathbb{M}_{n}, \mathfrak{D}_{n}, \mathfrak{D}_{n}^{+}, \mathbb{H}_{n}$ be the space of linear operators, density matrices, strictly positive density matrices and Hermitian matrices on $\mathcal{H}$, respectively. Let $\mathbb{M}_{n}^{0}$ and $\mathbb{H}_{n}^{0}$ be the space of traceless elements of $\mathbb{M}_{n}$ and $\mathbb{H}_{n}$, respectively. Denote the $n$-by- $n$ identity matrix by $\mathbb{I}_{n}$ (acting on $\mathcal{H}$ ); let $\mathcal{I}_{n}$ be the identity operator acting on $\mathbb{M}_{n}$. If the Hilbert space $\mathcal{H}=\mathcal{H}_{1} \otimes \mathcal{H}_{2} \otimes \cdots \otimes \mathcal{H}_{N}$, and $\mathcal{H}_{j}$ has the dimension $n_{j}$ (for $1 \leq j \leq N$ ), then the space of linear operators on $\mathcal{H}$ is denoted by $\mathbb{M}_{n_{1} \times n_{2} \times \cdots \times n_{N}}$; the same convention applies similarly to other spaces, e.g., $\mathbb{H}_{n_{1} \times n_{2} \times \cdots \times n_{N}}$. As a reminder, following the above notation convention, $\mathbb{H}_{n_{1} \times n_{2}}^{0} \neq \mathbb{H}_{n_{1}}^{0} \otimes \mathbb{H}_{n_{2}}^{0}$. 
Let $\langle\cdot, \cdot\rangle$ denote a generic inner product on $\mathbb{M}_{n}$; the Hilbert-Schmidt inner product is defined as $\langle A, B\rangle_{H S}:=\operatorname{Tr}\left(A^{\dagger} B\right)$. For any positive semidefinite operator $\mathscr{T}$ on $\mathbb{M}_{n}$, define the sesquilinear form $\langle A, B\rangle_{\mathscr{T}}:=\langle A, \mathscr{T}(B)\rangle_{H S}$ and the semi-norm $\|A\|_{\mathscr{T}}:=\sqrt{\langle A, A\rangle_{\mathscr{T}}}$ for all $A, B \in \mathbb{M}_{n}$; when $\mathscr{T}$ is strictly positive, the sesquilinear form becomes an inner product and the semi-norm becomes a norm.

For convenience, for any $A, B \in \mathbb{M}_{n}$, we denote

$$
A \# B:=L_{A} R_{B},
$$

where $L_{A}$ and $R_{B}$ are left and right multiplication of $A$ and $B$, respectively; in other words, $(A \# B)(X)=A X B$.

\subsection{Quantum $\chi_{\kappa}^{2}$ divergences}

Throughout this work, we consider the quantum $\chi_{\kappa}^{2}$ divergence, introduced in [21, Def. 1]. Let us introduce a set $\mathcal{K}$,

$$
\mathcal{K}:=\left\{\kappa:(0, \infty) \rightarrow(0, \infty) \mid-\kappa \text { is operator monotone, } \kappa(1)=1, x \kappa(x)=\kappa\left(x^{-1}\right)\right\} .
$$

As a remark, it is easy to check that $\kappa_{1 / 2}(x) \equiv x^{-1 / 2}$ is in the family $\mathcal{K}$.

Definition 2 (Quantum $\chi_{\kappa}^{2}$ divergence). For any $\kappa \in \mathcal{K}$, define the quantum $\chi_{\kappa}^{2}$ divergence between quantum states $\rho, \sigma \in \mathfrak{D}_{n}$ by

$$
\chi_{\kappa}^{2}(\rho \| \sigma):=\left\langle\rho-\sigma, \Omega_{\sigma}^{\kappa}(\rho-\sigma)\right\rangle_{H S}
$$

when $\operatorname{supp}(\rho) \subset \operatorname{supp}(\sigma)$; otherwise, set $\chi_{\kappa}^{2}(\rho \| \sigma)=\infty$. The operator $\Omega_{\sigma}^{\kappa}$ above is given by

$$
\Omega_{\sigma}^{\kappa}:=R_{\sigma}^{-1} \kappa\left(L_{\sigma} R_{\sigma}^{-1}\right) \equiv L_{\sigma}^{-1} \kappa\left(R_{\sigma} L_{\sigma}^{-1}\right) .
$$

The second equality comes from the assumption that $x \kappa(x)=\kappa\left(x^{-1}\right)$. As a remark, when $\sigma$ is not a full-rank density matrix, $\Omega_{\sigma}^{\kappa}$ can still be well-defined on the support of $\sigma$.

Essentially, the operator $\Omega_{\sigma}^{\kappa}$ is a non-commutative way to multiply $\sigma^{-1}$. Properties of the operator $\Omega_{\sigma}^{\kappa}$ will be further discussed in $\S 2.3$.

Next, let us introduce the non-commutative way to multiply $\sigma$. Define the weight operator $\Gamma_{\sigma}$

$$
\Gamma_{\sigma}:=\sigma^{1 / 2} \# \sigma^{1 / 2}
$$

Note that the operator $\Gamma_{\sigma}$ is completely positive, with the Kraus operator $\sigma^{1 / 2}$ and $\Omega_{\sigma}^{\kappa_{1 / 2}}=\left(\Gamma_{\sigma}\right)^{-1}$. For any $\kappa \in \mathcal{K}$, let us define a generalization of the operator $\Gamma_{\sigma}$

$$
\begin{aligned}
\mho_{\sigma}^{\kappa} & :=L_{\sigma} \kappa\left(L_{\sigma} R_{\sigma}^{-1}\right) \equiv L_{\sigma} \kappa\left(\sigma \# \sigma^{-1}\right) \\
& =\Gamma_{\sigma} \circ \Omega_{\sigma}^{\kappa} \circ \Gamma_{\sigma} .
\end{aligned}
$$

Notice that $\mho_{\sigma}^{\kappa_{1 / 2}}=\Gamma_{\sigma}$.

\subsection{Examples of $\kappa(x)$}

In this subsection, we provide three examples of $\kappa$ such that $\kappa \geq \kappa_{1 / 2}$ (satisfying one of the conditions in Theorem 1). More examples can be found in [9, Sec. 4.2] and [8, Sec. (III)].

Example 3 (Quantum $\chi_{\kappa_{\alpha}}^{2}$ divergence). An important family of the quantum $\chi_{\kappa}^{2}$ divergence is the quantum $\chi_{\kappa_{\alpha}}^{2}$ divergence, with the parameter $\alpha \in[0,1]$ and

$$
\kappa_{\alpha}(x)=\frac{1}{2}\left(x^{-\alpha}+x^{\alpha-1}\right) .
$$

(i) The case $\alpha=1 / 2$ is very special: $\kappa_{1 / 2}(x)=x^{-1 / 2}$ and $\Omega_{\sigma}^{\kappa_{1 / 2}}=\sigma^{-1 / 2} \# \sigma^{-1 / 2}$ is completely positive with the Kraus operator $\sigma^{-1 / 2}$. In fact, $\kappa_{1 / 2}$ is the only one in $\mathcal{K}$ such that for any $\sigma$, both $\Omega_{\sigma}^{\kappa}$ and $\left(\Omega_{\sigma}^{\kappa}\right)^{-1}$ are completely positive [9, Theorem 3.5]. 
(ii) We can immediately verify that $\kappa_{\alpha}=\kappa_{1-\alpha}$ and for any fixed $x \in(0, \infty), \kappa_{\alpha}(x)$ is monotonically decreasing with respect to $\alpha \in[0,1 / 2]$; thus $\kappa_{\alpha}(x) \geq \kappa_{1 / 2}(x)$.

More results about this family of the quantum $\chi_{\kappa_{\alpha}}^{2}$ divergence (also called mean $\alpha$-divergence) could be found in [21].

Example 4 (Wigner-Yanase-Dyson). Another family of $\kappa_{\beta}^{W Y D}$ (see e.g., $\left.[8,9]\right)$ corresponds to the Wigner-Yanase-Dyson metric, and it is parameterized by $\beta \in[-1,2]$,

$$
\kappa_{\beta}^{W Y D}(x):=\frac{1}{\beta(1-\beta)} \frac{\left(1-x^{\beta}\right)\left(1-x^{1-\beta}\right)}{(1-x)^{2}}, \quad x \in(0,1) \cup(1, \infty) .
$$

When $x=1, \kappa_{\beta}^{W Y D}(x)$ is simply set as 1 or is defined by taking the limit $x \rightarrow 1$ in the above equation. In general, finding all possible $\beta \in[-1,2]$ such that $\kappa_{\beta}^{W Y D} \geq \kappa_{1 / 2}$ seems to be slightly technical; however, at least, for a few special choices of $\beta$, e.g., when $\beta=1.5\left(\kappa_{1.5}^{W Y D}(x) \equiv\right.$ $\left.\kappa_{1 / 2}(x)+\frac{\left.x^{-1 / 2}(\sqrt{x}-1)^{4}\right)}{3(1-x)^{2}}\right)$ and $\beta=2\left(\kappa_{2}^{W Y D}(x) \equiv \frac{1+x}{2 x}\right)$ we can easily check that $\kappa_{\beta}^{W Y D} \geq \kappa_{1 / 2}$ for these two cases.

Example 5 (The largest possible $\kappa$ ). The largest $\kappa \in \mathcal{K}$ is $\kappa_{\max }:=\frac{1+x}{2 x}$ (see e.g., [8, Eq. (11)]). It is obvious that $\kappa_{\max } \geq \kappa_{1 / 2}$.

As a remark, $\kappa_{2}^{W Y D}$ in the family of Wigner-Yanase-Dyson metric is exactly the maximum one.

\subsection{Basic properties of operators $\Omega_{\sigma}^{\kappa}$ and $\mho_{\sigma}^{\kappa}$}

We list without proof some elementary while useful properties of the operator $\Omega_{\sigma}^{\kappa}$. Recall the assumption that $x \kappa(x)=\kappa\left(x^{-1}\right)$, which is used below in the proof of $\Omega_{\sigma}^{\kappa}$ being Hermitianpreserving.

Lemma 6. Suppose $\sigma \in \mathfrak{D}_{n}^{+}$and its eigenvalue decomposition $\sigma=\sum_{j=1}^{n} s_{j}\left|s_{j}\right\rangle\left\langle s_{j}\right|$. Then

(i) The operator $\Omega_{\sigma}^{\kappa}$ can be decomposed as

$$
\Omega_{\sigma}^{\kappa}=\sum_{j, m=1}^{n} \kappa\left(\frac{s_{j}}{s_{m}}\right) \frac{1}{s_{m}}\left|s_{j}\right\rangle\left\langle s_{j}|\#| s_{m}\right\rangle\left\langle s_{m}\right|
$$

For any Hermitian matrix $A \in \mathbb{M}_{n}$,

$$
\langle A, A\rangle_{\Omega_{\sigma}^{\kappa}} \equiv\left\langle A, \Omega_{\sigma}^{\kappa}(A)\right\rangle_{H S}=\sum_{j, m=1}^{n} \kappa\left(\frac{s_{j}}{s_{m}}\right) \frac{1}{s_{m}}\left|\left\langle s_{j}|A| s_{m}\right\rangle\right|^{2} \geq 0 .
$$

Thus, $\Omega_{\sigma}^{\kappa}$ is a strictly positive operator with respect to the Hilbert-Schmidt inner product, and the inner product $\langle\cdot, \cdot\rangle_{\Omega_{\sigma}^{\kappa}}$ is well-defined.

(ii) $\Omega_{\sigma}^{\kappa}$ is Hermitian-preserving.

(iii) We have $\Omega_{\sigma}^{\kappa}(\sigma)=\mathbb{I}_{n}$. Thus for any $A \in \mathbb{M}_{n}$,

$$
\langle A, \sigma\rangle_{\Omega_{\sigma}^{\kappa}}=\left\langle A, \mathbb{I}_{n}\right\rangle_{H S}, \quad\langle\sigma, A\rangle_{\Omega_{\sigma}^{\kappa}}=\left\langle\mathbb{I}_{n}, A\right\rangle_{H S}=\operatorname{Tr}(A) .
$$

In particular, for any density matrix $\rho \in \mathfrak{D}_{n},\langle\rho, \sigma\rangle_{\Omega_{\sigma}^{\kappa}}=\langle\sigma, \rho\rangle_{\Omega_{\sigma}^{\kappa}}=1$.

Then let us consider the properties of $\Omega_{\sigma}^{\kappa}$ for a composite system.

Lemma 7. (i) Consider $\sigma_{1} \in \mathfrak{D}_{n_{1}}^{+}$and $\sigma_{2} \in \mathfrak{D}_{n_{2}}^{+}$. Then for any $A \in \mathbb{M}_{n_{1}}$ and $B \in \mathbb{M}_{n_{2}}$, we have

$$
\Omega_{\sigma_{1} \otimes \sigma_{2}}^{\kappa}\left(A \otimes \sigma_{2}\right)=\Omega_{\sigma_{1}}^{\kappa}(A) \otimes \mathbb{I}_{n_{2}} \quad \Omega_{\sigma_{1} \otimes \sigma_{2}}^{\kappa}\left(\sigma_{1} \otimes B\right)=\mathbb{I}_{n_{1}} \otimes \Omega_{\sigma_{2}}^{\kappa}(B) .
$$


(ii) $\kappa_{1 / 2}$ is the only one in $\mathcal{K}$ such that for all $\sigma_{1} \in \mathfrak{D}_{n_{1}}^{+}$and $\sigma_{2} \in \mathfrak{D}_{n_{2}}^{+}$, we have

$$
\Omega_{\sigma_{1} \otimes \sigma_{2}}^{\kappa}=\Omega_{\sigma_{1}}^{\kappa} \otimes \Omega_{\sigma_{2}}^{\kappa} .
$$

Proof. Let us decompose $\sigma_{1}=\sum_{j=1}^{n_{1}} \lambda_{j}\left|\psi_{j}\right\rangle\left\langle\psi_{j}\right|$ and $\sigma_{2}=\sum_{m=1}^{n_{2}} \mu_{m}\left|\phi_{m}\right\rangle\left\langle\phi_{m}\right|$, then $\sigma_{1} \otimes \sigma_{2}$ has an eigenvalue decomposition $\sigma_{1} \otimes \sigma_{2}=\sum_{j, m} \lambda_{j} \mu_{m}\left|\psi_{j}\right\rangle\left\langle\psi_{j}|\otimes| \phi_{m}\right\rangle\left\langle\phi_{m}\right|$.

(i) By the decomposition of the operator $\Omega_{(\cdot)}^{k}$ in $(15)$,

$$
\Omega_{\sigma_{1} \otimes \sigma_{2}}^{\kappa}=\sum_{j_{1}, j_{2}, m_{1}, m_{2}} \kappa\left(\frac{\lambda_{j_{1}} \mu_{m_{1}}}{\lambda_{j_{2}} \mu_{m_{2}}}\right) \frac{1}{\lambda_{j_{2}} \mu_{m_{2}}}\left(\left|\psi_{j_{1}}\right\rangle\left\langle\psi_{j_{1}}|\otimes| \phi_{m_{1}}\right\rangle\left\langle\phi_{m_{1}}\right|\right) \#\left(\left|\psi_{j_{2}}\right\rangle\left\langle\psi_{j_{2}}|\otimes| \phi_{m_{2}}\right\rangle\left\langle\phi_{m_{2}}\right|\right) .
$$

Then by direct calculation,

$$
\begin{aligned}
& \Omega_{\sigma_{1} \otimes \sigma_{2}}^{\kappa}\left(A \otimes \sigma_{2}\right) \\
= & \sum_{j_{1}, j_{2}, m_{1}, m_{2}} \kappa\left(\frac{\lambda_{j_{1}} \mu_{m_{1}}}{\lambda_{j_{2}} \mu_{m_{2}}}\right) \frac{1}{\lambda_{j_{2}} \mu_{m_{2}}} \mu_{m_{1}} \delta_{m_{1}, m_{2}}\left(\left|\psi_{j_{1}}\right\rangle\left\langle\psi_{j_{1}}|A| \psi_{j_{2}}\right\rangle\left\langle\psi_{j_{2}}\right|\right) \otimes\left|\phi_{m_{1}}\right\rangle\left\langle\phi_{m_{1}}\right| \\
= & \sum_{j_{1}, j_{2}, m_{1}} \kappa\left(\frac{\lambda_{j_{1}}}{\lambda_{j_{2}}}\right) \frac{1}{\lambda_{j_{2}}}\left(\left|\psi_{j_{1}}\right\rangle\left\langle\psi_{j_{1}}|A| \psi_{j_{2}}\right\rangle\left\langle\psi_{j_{2}}\right|\right) \otimes\left|\phi_{m_{1}}\right\rangle\left\langle\phi_{m_{1}}\right| \\
= & \Omega_{\sigma_{1}}^{\kappa}(A) \otimes \mathbb{I}_{n_{2}} .
\end{aligned}
$$

The other case can be similarly proved.

(ii) When $\kappa=\kappa_{1 / 2}$, by the fact that $\Omega_{\sigma}^{\kappa_{1 / 2}}=\left(\Gamma_{\sigma}\right)^{-1}$, we can immediately see the tensorization (19). As for the other direction, from the assumption that (19) holds and after some straightforward simplification, one could obtain that $\kappa\left(\frac{\lambda_{j_{1}} \mu_{m_{1}}}{\lambda_{j_{2}} \mu_{m_{2}}}\right)=\kappa\left(\frac{\lambda_{j_{1}}}{\lambda_{j_{2}}}\right) \kappa\left(\frac{\mu_{m_{1}}}{\mu_{m_{2}}}\right)$, for all indices $j_{1}, j_{2}, m_{1}, m_{2}$. Since $\sigma_{1}$ and $\sigma_{2}$ are arbitrary density matrices, we have $\kappa(x y)=\kappa(x) \kappa(y)$ for all $x, y>0$; in particular, $1=\kappa(1)=\kappa(x) \kappa\left(x^{-1}\right)$. Since $\kappa \in \mathcal{K}$, we also have $x \kappa(x)=\kappa\left(x^{-1}\right)$, which leads into $\kappa(x)=x^{-1 / 2}=\kappa_{1 / 2}$.

Similarly, we list without proof the following properties of $\mho_{\sigma}^{\kappa}$; all properties can be easily verified by the definition of $\mho_{\sigma}^{\kappa}$ in $(11)$.

Lemma 8 (Operator $\mho_{\sigma}^{\kappa}$ ). Suppose $\sigma \in \mathfrak{D}_{n}^{+}$and its eigenvalue decomposition $\sigma=\sum_{j=1}^{n} s_{j}\left|s_{j}\right\rangle\left\langle s_{j}\right|$. Then

(i) the operator $\mho_{\sigma}^{\kappa}$ for any $\kappa \in \mathcal{K}$ has a decomposition

$$
\mho_{\sigma}^{\kappa}=\sum_{j, m} s_{j} \kappa\left(\frac{s_{j}}{s_{m}}\right)\left|s_{j}\right\rangle\left\langle s_{j}|\#| s_{m}\right\rangle\left\langle s_{m}\right|
$$

thus $\mho_{\sigma}^{\kappa}$ is strictly positive with respect to the Hilbert-Schmidt inner product;

(ii) the operator $\mho_{\sigma}^{\kappa}$ is Hermitian-preserving;

(iii) $\mho_{\sigma}^{\kappa}\left(\mathbb{I}_{n}\right)=\sigma$.

\subsection{Eigenvalue formalism of SDPI constants}

The eigenvalue formalism of the quantum contraction coefficient can be found in e.g. [8, 12, 20]; the classical analogous result can be found in e.g., $[5,18]$. In this subsection, we concisely present this formalism, for the sake of completeness.

Let us consider the ratio in the SDPI constant.

$$
\frac{\chi_{\kappa}^{2}(\mathcal{E}(\rho) \| \mathcal{E}(\sigma))}{\chi_{\kappa}^{2}(\rho \| \sigma)}=\frac{\left\langle\rho-\sigma, \mathcal{E}^{\dagger} \circ \Omega_{\mathcal{E}(\sigma)}^{\kappa} \circ \mathcal{E}(\rho-\sigma)\right\rangle_{H S}}{\left\langle\rho-\sigma, \Omega_{\sigma}^{\kappa}(\rho-\sigma)\right\rangle_{H S}}=\frac{\left\langle\rho-\sigma, \Upsilon_{\mathcal{E}, \sigma}^{\kappa}(\rho-\sigma)\right\rangle_{\Omega_{\sigma}^{\kappa}}}{\langle\rho-\sigma, \rho-\sigma\rangle_{\Omega_{\sigma}^{\kappa}}}
$$


where we introduce

$$
\Upsilon_{\mathcal{E}, \sigma}^{\kappa}:=\left(\Omega_{\sigma}^{\kappa}\right)^{-1} \circ \mathcal{E}^{\dagger} \circ \Omega_{\mathcal{E}(\sigma)}^{\kappa} \circ \mathcal{E} .
$$

Here are some properties of the operator $\Upsilon_{\mathcal{E}, \sigma}^{\kappa}$.

Lemma 9. Assume that $\sigma, \mathcal{E}(\sigma) \in \mathfrak{D}_{n}^{+}$.

(i) The operator $\Upsilon_{\mathcal{E}, \sigma}^{\kappa}$ is positive semidefinite with respect to the inner product $\langle\cdot, \cdot\rangle_{\Omega_{\sigma}^{\kappa}}$.

(ii) $\Upsilon_{\mathcal{E}, \sigma}^{\kappa}(\sigma)=\sigma$.

(iii) $\Upsilon_{\mathcal{E}, \sigma}^{\kappa}$ is Hermitian perserving.

(iv) For any $A \in \mathbb{M}_{n}$, we have

$$
\left\langle A, \Upsilon_{\mathcal{E}, \sigma}^{\kappa}(A)\right\rangle_{\Omega_{\sigma}^{\kappa}} \leq\langle A, A\rangle_{\Omega_{\sigma}^{\kappa}}
$$

Therefore, the eigenvalue of $\Upsilon_{\mathcal{E}, \sigma}^{\kappa}$ is bounded above by 1.

Proof. Part (i) is obvious from (22) and Lemma 6 (i). Part (ii) can be verified directly by Lemma 6 (iii) and the fact that $\mathcal{E}$ is trace-preserving (or equivalently $\mathcal{E}^{\dagger}$ is unital). As for part (iii), since the quantum channel $\mathcal{E}$ is completely positive, it is thus also Hermitian-preserving; so is $\mathcal{E}^{\dagger}$. By Lemma 6 (ii), $\Omega_{\sigma}^{\kappa}$ is Hermitian-preserving, thus so is $\left(\Omega_{\sigma}^{\kappa}\right)^{-1}$. Finally, since the composition of two Hermitian-preserving operators is also Hermitian-preserving, we conclude that $\Upsilon_{\mathcal{E}, \sigma}^{\kappa}$ is Hermitianpreserving. Part (iv) is essentially the data processing inequality; see e.g. [12, Thm. II.14] and [21, Thm. 4] for the proof.

Then

$$
\begin{aligned}
\eta_{\chi_{\kappa}^{2}}(\mathcal{E}, \sigma) & \stackrel{(3)}{=} \sup _{\rho \in \mathfrak{D}_{n}: \rho \neq \sigma} \frac{\chi_{\kappa}^{2}(\mathcal{E}(\rho) \| \mathcal{E}(\sigma))}{\chi_{\kappa}^{2}(\rho \| \sigma)} \\
& \stackrel{(21)}{=} \sup _{\rho \geq 0: \rho \neq \sigma, \operatorname{Tr}(\rho)=1} \frac{\left\langle\rho-\sigma, \Upsilon_{\mathcal{E}, \sigma}^{\kappa}(\rho-\sigma)\right\rangle_{\Omega_{\sigma}^{\kappa}}}{\langle\rho-\sigma, \rho-\sigma\rangle_{\Omega_{\sigma}^{\kappa}}} \\
& =\sup _{A \in \mathbb{H}_{n}^{0}: A \neq 0} \frac{\left\langle A, \Upsilon_{\mathcal{E}, \sigma}^{\kappa}(A)\right\rangle_{\Omega_{\sigma}^{\kappa}}}{\langle A, A\rangle_{\Omega_{\sigma}^{\kappa}}} .
\end{aligned}
$$

As one might observe, the last equation is closely connected to the eigenvalue formalism of the operator $\Upsilon_{\mathcal{E}, \sigma}^{\kappa}$, which is stated in the following lemma.

Lemma 10. For $\sigma \in \mathfrak{D}_{n}^{+}$and $\kappa \in \mathcal{K}$ and for any quantum channel $\mathcal{E}$ such that $\mathcal{E}(\sigma) \in \mathfrak{D}_{n}^{+}$, let $\lambda_{2}\left(\Upsilon_{\mathcal{E}, \sigma}^{\kappa}\right)$ be the second largest eigenvalue of $\Upsilon_{\mathcal{E}, \sigma}^{\kappa}$ (defined in (22)). Then

$$
\eta_{\chi_{\kappa}^{2}}(\mathcal{E}, \sigma)=\lambda_{2}\left(\Upsilon_{\mathcal{E}, \sigma}^{\kappa}\right) .
$$

Proof. Since $\Upsilon_{\mathcal{E}, \sigma}^{\kappa}$ is positive semidefinite with respect to the inner product $\langle\cdot, \cdot\rangle_{\Omega_{\sigma}^{\kappa}}$ from Lemma 9 (i), it admits a spectral decomposition with $\Upsilon_{\mathcal{E}, \sigma}^{\kappa}\left(V_{j}\right)=\theta_{j} V_{j}, \theta_{j} \geq 0$, where $j=1,2, \cdots, n^{2}$ and $\left\{V_{j}\right\}_{j=1}^{n^{2}}$ is an orthonormal basis in the Hilbert space $\left(\mathbb{M}_{n},\langle\cdot, \cdot\rangle_{\Omega_{\sigma}^{\kappa}}\right)$. Note that $\sigma$ is always an eigenvector of $\Upsilon_{\mathcal{E}, \sigma}^{\kappa}$ from Lemma 9 (ii); without loss of generality, let $V_{1}=\sigma$ and $\theta_{1}=1$. By the orthogonality of $\left\{V_{j}\right\}_{j}$, we know $0=\left\langle\sigma, V_{j}\right\rangle_{\Omega_{\sigma}^{\kappa}}=\operatorname{Tr}\left(V_{j}\right)$ for $j \geq 2$. By Lemma 9 (iv), $\theta_{j} \leq 1$ for all $1 \leq j \leq n^{2}$; thus without loss of generality, assume $\theta_{j}$ are listed in descending order and hence $\lambda_{2}\left(\Upsilon_{\mathcal{E}, \sigma}^{\kappa}\right)=\theta_{2}$. By rewriting $A=\sum_{j=2}^{n^{2}} c_{j} V_{j}$ in (23) where $c_{j} \in \mathbb{C}$, we immediately know that $\eta_{\chi_{\kappa}^{2}}(\mathcal{E}, \sigma) \leq \lambda_{2}\left(\Upsilon_{\mathcal{E}, \sigma}^{\kappa}\right)$.

By the fact that $\Upsilon_{\mathcal{E}, \sigma}^{\kappa}$ is Hermitian-preserving (see Lemma 9 (iii)), $V_{2}^{\dagger}$ is also an eigenvector associated with the eigenvalue $\lambda_{2}\left(\Upsilon_{\mathcal{E}, \sigma}^{\kappa}\right)$. Then we choose $A \in \mathbb{H}_{n}^{0}$ in $(23)$ by $\frac{V_{2}+V_{2}^{\dagger}}{2}$ or $\frac{V_{2}-V_{2}^{\dagger}}{2 i}$. Note that such an $A$ is also an eigenvector of $\Upsilon_{\mathcal{E}, \sigma}^{\kappa}$ with the eigenvalue $\lambda_{2}\left(\Upsilon_{\mathcal{E}, \sigma}^{\kappa}\right)$. Then $\eta_{\chi_{\kappa}^{2}}(\mathcal{E}, \sigma) \geq$ $\left\langle A, \Upsilon_{\mathcal{E}, \sigma}^{\kappa}(A)\right\rangle_{\Omega_{\sigma}^{\kappa}} /\langle A, A\rangle_{\Omega_{\sigma}^{\kappa}}=\lambda_{2}\left(\Upsilon_{\mathcal{E}, \sigma}^{\kappa}\right)$. 


\subsection{Another variational formalism of SDPI constants}

Recall the definition of the operator $\mho_{\sigma}^{\kappa}$ from (12). In Lemma 11 below, we provide another variational characterization of the SDPI constant; essentially, it follows from the connection between the eigenvalue formalism (as discussed in the last subsection) and the corresponding singular value formalism. Its classical version is well-known and can be found in e.g. the proof of [18, Thm. III.2]. This idea for quantum $\chi_{\kappa}^{2}$ divergences has appeared implicitly in [21, Thm. 9]; however, we don't assume $\sigma$ to be the stationary state of the quantum channel herein, compared with [21].

Lemma 11. Assume that quantum states $\sigma, \mathcal{E}(\sigma) \in \mathfrak{D}_{n}^{+}$. For any $\kappa \in \mathcal{K}$,

$$
\sqrt{\eta_{\chi_{\kappa}^{2}}(\mathcal{E}, \sigma)}=\max _{F, G}\left|\langle\mathscr{K}(F), G\rangle_{\mho_{\mathcal{E}(\sigma)}^{\kappa}}\right|,
$$

where the operator $\mathscr{K}$ is defined by

$$
\mathscr{K}:=\Gamma_{\mathcal{E}(\sigma)}^{-1} \circ \mathcal{E} \circ \Gamma_{\sigma},
$$

and the maximum is taken over all $F, G \in \mathbb{M}_{n}$ such that

$$
\left\langle\mathbb{I}_{n}, F\right\rangle_{\mho_{\sigma}^{\kappa}}=\left\langle\mathbb{I}_{n}, G\right\rangle_{\mho_{\mathcal{E}(\sigma)}^{\kappa}}=0, \quad\|F\|_{\mho_{\sigma}^{\kappa}}=\|G\|_{\mho_{\mathcal{E}(\sigma)}^{\kappa}}=1 .
$$

Proof of Lemma 11. First, we rewrite Lemma 10 in the language of the relative density (whose classical analog is the Radon-Nikodym derivative); specifically, to get the third equality below, $A$ is replaced by $\Gamma_{\sigma}(A)$. By Lemma 10,

$$
\begin{aligned}
& \eta_{\chi_{\kappa}^{2}}(\mathcal{E}, \sigma)=\sup _{A \in \mathbb{M}_{n}^{0}, A \neq 0} \frac{\left\langle A, \Upsilon_{\mathcal{E}, \sigma}^{\kappa}(A)\right\rangle_{\Omega_{\sigma}^{\kappa}}}{\langle A, A\rangle_{\Omega_{\sigma}^{\kappa}}}=\sup _{A \in \mathbb{M}_{n}^{0}, A \neq 0} \frac{\left\langle\mathcal{E}(A), \Omega_{\mathcal{E}(\sigma)}^{\kappa} \circ \mathcal{E}(A)\right\rangle_{H S}}{\left\langle A, \Omega_{\sigma}^{\kappa}(A)\right\rangle_{H S}} \\
&= \sup _{A \neq 0,\left\langle\mathbb{I}_{n}, A\right\rangle_{\mho_{\sigma}^{\kappa}=0}} \frac{\left\langle\mathcal{E} \circ \Gamma_{\sigma}(A), \Omega_{\mathcal{E}(\sigma)}^{\kappa} \circ \mathcal{E} \circ \Gamma_{\sigma}(A)\right\rangle_{H S} \stackrel{(12)}{=} \sup _{A \neq 0,\left\langle\mathbb{I}_{n}, A\right\rangle_{\mho_{\sigma}^{\kappa}}=0} \frac{\left\langle\mathscr{K}(A), \mho \mho_{\mathcal{E}(\sigma)}^{\kappa} \circ \mathscr{K}(A)\right\rangle_{H S}}{\left.\langle A, A\rangle_{\mho_{\sigma}^{\kappa}}(A), \Omega_{\sigma}^{\kappa} \circ \Gamma_{\sigma}(A)\right\rangle_{H S}}}{\left\langle A,\left(\mho_{\sigma}^{\kappa}\right)^{-1} \circ \mathscr{K}^{\dagger} \circ \mho_{\mathcal{E}(\sigma)}^{\kappa} \circ \mathscr{K}(A)\right\rangle_{\mho_{\sigma}^{\kappa}}} . \\
&=\sup _{A \neq 0,\left\langle\mathbb{I}_{n}, A\right\rangle_{\mho_{\sigma}^{\kappa}=0}} \frac{\langle A, A\rangle_{\mho_{\sigma}^{\kappa}}}{\langle A}
\end{aligned}
$$

As for the operator $\mathscr{K}$, it can be straightforwardly checked that

- $\mathscr{K}$ is completely positive and unital $\left(\mathscr{K}\left(\mathbb{I}_{n}\right)=\mathbb{I}_{n}\right)$.

- $\mathscr{K}^{\dagger}=\Gamma_{\sigma} \circ \mathcal{E}^{\dagger} \circ \Gamma_{\mathcal{E}(\sigma)}^{-1}$ is completely positive, trace-preserving, and $\mathscr{K}^{\dagger}(\mathcal{E}(\sigma))=\sigma$.

- Consider the following two Hilbert spaces $\mathscr{H}_{1}$ and $\mathscr{H}_{2}$,

$$
\begin{aligned}
& \mathscr{H}_{1}:=\left\{A \in \mathbb{M}_{n}:\left\langle\mathbb{I}_{n}, A\right\rangle_{\mho_{\sigma}^{\kappa}}=0\right\}, \text { equipped with the inner product }\langle\cdot, \cdot\rangle_{\mho_{\sigma}^{\kappa}} ; \\
& \mathscr{H}_{2}:=\left\{A \in \mathbb{M}_{n}:\left\langle\mathbb{I}_{n}, A\right\rangle_{\mho_{\mathcal{E}(\sigma)}^{\kappa}}=0\right\}, \text { equipped with the inner product }\langle\cdot, \cdot\rangle_{\mho_{\mathcal{E}(\sigma)}^{\kappa}} .
\end{aligned}
$$

Then we can readily verify that $\mathscr{K}$ is an operator from $\mathscr{H}_{1}$ to $\mathscr{H}_{2}$, i.e., if $\left\langle\mathbb{I}_{n}, A\right\rangle_{\mho_{\sigma}^{\kappa}}=0$, then $\left\langle\mathbb{I}_{n}, \mathscr{K}(A)\right\rangle_{\mho_{\mathcal{E}(\sigma)}^{\kappa}}=0$. The dual operator of $\mathscr{K}$, denoted by $\widetilde{\mathscr{K}}$, maps from $\mathscr{H}_{2}$ to $\mathscr{H}_{1}$ and it is explicitly given by $\widetilde{\mathscr{K}}=\left(\mho_{\sigma}^{\kappa}\right)^{-1} \circ \mathscr{K}^{\dagger} \circ \mho_{\mathcal{E}(\sigma)}^{\kappa}$.

Then, we have

$$
\eta_{\chi_{\kappa}^{2}}(\mathcal{E}, \sigma)=\sup _{A \neq 0, A \in \mathscr{H}_{1}} \frac{\langle A, \widetilde{\mathscr{K}} \circ \mathscr{K}(A)\rangle_{\mho_{\sigma}^{\kappa}}}{\langle A, A\rangle_{\mho_{\sigma}^{\kappa}}} .
$$

Let us denote the SVD decomposition of $\widetilde{\mathscr{K}}$ by $\widetilde{\mathscr{K}}(\cdot)=\sum_{j} a_{j} \phi_{j}\left\langle\varphi_{j}, \cdot\right\rangle_{\mho_{\mathcal{E}(\sigma)}^{\kappa}}$ where $a_{j} \geq 0$, $\left\{\phi_{j}\right\}_{j}$ and $\left\{\varphi_{j}\right\}_{j}$ are orthonormal basis of $\mathscr{H}_{1}$ and $\mathscr{H}_{2}$ respectively. Then, easily we know $\mathscr{K}(\cdot)=$ $\sum_{j} a_{j} \varphi_{j}\left\langle\phi_{j}, \cdot\right\rangle_{\mho_{\sigma}^{\kappa}}$ and that $\widetilde{\mathscr{K}} \circ \mathscr{K}(\cdot)=\sum_{j} a_{j}^{2} \phi_{j}\left\langle\phi_{j}, \cdot\right\rangle_{\mho_{\sigma}^{\kappa}}$. Then $\eta_{\chi_{\kappa}^{2}}(\mathcal{E}, \sigma)$ is simply the largest value of $a_{j}^{2}$; namely, $\sqrt{\eta_{\chi_{\kappa}^{2}}(\mathcal{E}, \sigma)}$ is the largest singular value of $\mathscr{K}$, and the result in Lemma 11 follows immediately. 


\subsection{Comparison of SDPI constants}

First, we provide a uniform lower bound of $\sqrt{\eta_{\chi_{\kappa}^{2}}(\mathcal{E}, \sigma)}$ for any $\kappa \in \mathcal{K}$ in terms of $\eta_{\chi_{\kappa_{1 / 2}}^{2}}(\mathcal{E}, \sigma)$ in Lemma 12, which is a new result to the best of our knowledge. One of our corollaries in (30) can also be derived by [8, Thm. 4.4] and [8, Thm. 5.3]. However, our approach to show (30) is different from [8]: their result comes from comparing the contraction coefficient $\eta_{\chi_{\kappa}^{2}}(\mathcal{E})$ with $\eta_{\operatorname{Tr}}(\mathcal{E})$ (the contraction coefficient for trace norm); we use the SDPI constant of the Petz recovery map as the bridge. Second, we consider quantum-classical (QC) channels and provide the ordering of SDPI constants for different $\kappa$ in Lemma 14; similar results have appeared in [8, Prop. 5.5] for contraction coefficients.

Lemma 12. For any quantum channel $\mathcal{E}$ and quantum state $\sigma \in \mathfrak{D}_{n}^{+}$such that $\mathcal{E}(\sigma) \in \mathfrak{D}_{n}^{+}$, we have

$$
\eta_{\chi_{\kappa_{1 / 2}^{2}}^{2}}(\mathcal{E}, \sigma) \leq \sqrt{\eta_{\chi_{\kappa}^{2}}(\mathcal{E}, \sigma) \eta_{\chi_{\kappa}^{2}}\left(\mathcal{R}_{\mathcal{E}, \sigma}, \mathcal{E}(\sigma)\right)} \leq \sqrt{\eta_{\chi_{\kappa}^{2}}(\mathcal{E}, \sigma)}
$$

where $\mathcal{R}_{\mathcal{E}, \sigma}$ is the Petz recovery map, defined by

$$
\mathcal{R}_{\mathcal{E}, \sigma}(A):=\sigma^{1 / 2} \mathcal{E}^{\dagger}\left(\mathcal{E}(\sigma)^{-1 / 2} A \mathcal{E}(\sigma)^{-1 / 2}\right) \sigma^{1 / 2} \equiv \Gamma_{\sigma} \circ \mathcal{E}^{\dagger} \circ \Gamma_{\mathcal{E}(\sigma)}^{-1}(A), \quad \forall A \in \mathbb{M}_{n}
$$

mapping $\mathcal{E}(\sigma)$ to $\sigma$.

The followings are immediate consequences of the lemma above.

Corollary 13. Under the same assumption as in Lemma 12,

(i) The SDPI constant associated with $\kappa_{1 / 2}$ for the pair $(\mathcal{E}, \sigma)$ equals the SDPI constant for the recovery map pair $\left(\mathcal{R}_{\mathcal{E}, \sigma}, \mathcal{E}(\sigma)\right)$, that is to say,

$$
\eta_{\chi_{\kappa_{1 / 2}}^{2}}(\mathcal{E}, \sigma)=\eta_{\chi_{\kappa_{1 / 2}}^{2}}\left(\mathcal{R}_{\mathcal{E}, \sigma}, \mathcal{E}(\sigma)\right) .
$$

(ii) Further assume that for any $\sigma \in \mathfrak{D}_{n}^{+}$, we have $\mathcal{E}(\sigma) \in \mathfrak{D}_{n}^{+}$. Then, for the contraction coefficient of the quantum channel $\mathcal{E}$, we have

$$
\eta_{\chi_{\kappa_{1 / 2}^{2}}}(\mathcal{E}) \leq \sqrt{\eta_{\chi_{\kappa}^{2}}(\mathcal{E})}
$$

Proof. The first part comes from letting $\kappa=\kappa_{1 / 2}$ in (28) and the fact that the Petz recovery map of $\mathcal{R}_{\mathcal{E}, \sigma}$ is exactly the channel $\mathcal{E}$; the second part comes from taking the supremum over all $\sigma \in \mathfrak{D}_{n}^{+}$.

Proof of Lemma 12. It is straightforward to verify that $\mathcal{R}_{\mathcal{E}, \sigma}$, defined in (29), is a bona-fide quantum channel, mapping the quantum state $\mathcal{E}(\sigma)$ back to $\sigma$. We can easily verify by definition $(22)$ and (29) that

$$
\Upsilon_{\mathcal{E}, \sigma}^{\kappa_{1 / 2}}=\mathcal{R}_{\mathcal{E}, \sigma} \circ \mathcal{E}
$$

Recall from Lemma 10 that there exists a $\lambda_{2} \equiv \lambda_{2}\left(\Upsilon_{\mathcal{E}, \sigma}^{\kappa_{1 / 2}}\right)=\eta_{\chi_{\kappa_{1 / 2}^{2}}^{2}}(\mathcal{E}, \sigma)$ and a traceless Hermitian matrix $V \in \mathbb{H}_{n}^{0}$ such that $\Upsilon_{\mathcal{E}, \sigma}^{\kappa_{1 / 2}}(V)=\lambda_{2} V$. Let $\widetilde{V}:=\mathcal{E}(V) \in \mathbb{H}_{n}^{0}$. Then

$$
\begin{aligned}
\left(\eta_{\chi_{\kappa_{1} / 2}^{2}}(\mathcal{E}, \sigma)\right)^{2} & =\lambda_{2}^{2}=\frac{\left\langle\Upsilon_{\mathcal{E}, \sigma}^{\kappa_{1 / 2}}(V), \Upsilon_{\mathcal{E}, \sigma}^{\kappa_{1 / 2}}(V)\right\rangle_{\Omega_{\sigma}^{\kappa}}}{\langle V, V\rangle_{\Omega_{\sigma}^{\kappa}}} \\
& \stackrel{(31)}{=} \frac{\left\langle\mathcal{R}_{\mathcal{E}, \sigma}(\widetilde{V}), \Omega_{\sigma}^{\kappa} \circ \mathcal{R}_{\mathcal{E}, \sigma}(\widetilde{V})\right\rangle_{H S}}{\langle V, V\rangle_{\Omega_{\sigma}^{\kappa}}} \\
& =\frac{\left\langle\mathcal{R}_{\mathcal{E}, \sigma}(\widetilde{V}), \Omega_{\mathcal{R}_{\mathcal{E}, \sigma}^{\kappa}(\mathcal{E}(\sigma))} \circ \mathcal{R}_{\mathcal{E}, \sigma}(\widetilde{V})\right\rangle_{H S}}{\left\langle\widetilde{V}, \Omega_{\mathcal{E}(\sigma)}^{\kappa}(\widetilde{V})\right\rangle_{H S}} \\
& \leq \eta_{\chi_{\kappa}^{2}}\left(\mathcal{R}_{\mathcal{E}, \sigma}, \mathcal{E}(\sigma)\right) \eta_{\chi_{\kappa}^{2}}(\mathcal{E}, \sigma)
\end{aligned}
$$


The inequality in the last step follows from Lemma 10. Hence, we have proved the first inequality in (28); the second inequality follows immediately from the data processing inequality of the quantum $\chi_{\kappa}^{2}$ divergence.

Next, we consider any quantum-classical (QC) channel $\mathcal{E}$, which refers to a physical process in which one first performs a measurement according to a POVM $\left\{F_{j}\right\}_{j=1}^{n}\left(F_{j} \in \mathbb{M}_{n}\right.$ are positive semidefinite and $\sum_{j=1}^{n} F_{j}=\mathbb{I}_{n}$ ); then based on the measurement outcome, one prepares a pure state, selected from a set $\left\{\psi_{j}\right\}_{j=1}^{n}$ which also forms an orthonormal basis of $\mathcal{H}$. More specifically,

$$
\mathcal{E}(A)=\sum_{j=1}^{n} \operatorname{Tr}\left(F_{j} A\right)\left|\psi_{j}\right\rangle\left\langle\psi_{j}\right|, \quad \forall A \in \mathbb{M}_{n} .
$$

Define a ratio $\mathrm{R}_{\mathcal{E}, \sigma}^{\kappa}$ on $\mathbb{H}_{n}^{0}$ by

$$
\mathrm{R}_{\mathcal{E}, \sigma}^{\kappa}(A):=\frac{\left\langle\mathcal{E}(A), \Omega_{\mathcal{E}(\sigma)}^{\kappa} \circ \mathcal{E}(A)\right\rangle_{H S}}{\left\langle A, \Omega_{\sigma}^{\kappa}(A)\right\rangle_{H S}} \equiv \frac{\left\langle A, \Upsilon_{\mathcal{E}, \sigma}^{\kappa}(A)\right\rangle_{\Omega_{\sigma}^{\kappa}}}{\langle A, A\rangle_{\Omega_{\sigma}^{\kappa}}}, \quad \text { for } A \in \mathbb{H}_{n}^{0}, A \neq 0 .
$$

Lemma 14. Suppose $\kappa \geq \kappa_{1 / 2}, \mathcal{E}$ is a $Q C$ channel with $F_{j} \neq 0$ for all $1 \leq j \leq n$ and $\sigma \in \mathfrak{D}_{n}^{+}$. Then

$$
\mathrm{R}_{\mathcal{E}, \sigma}^{\kappa}(A) \leq \mathrm{R}_{\mathcal{E}, \sigma}^{\kappa_{1 / 2}}(A), \quad \forall A \in \mathbb{H}_{n}^{0}, A \neq 0
$$

Consequently, we have

$$
\eta_{\chi_{\kappa}^{2}}(\mathcal{E}, \sigma) \leq \eta_{\chi_{\kappa_{1 / 2}}^{2}}(\mathcal{E}, \sigma)
$$

Proof. By (32) and (16), we can readily calculate that

$$
\left\langle\mathcal{E}(A), \Omega_{\mathcal{E}(\sigma)}^{\kappa}(\mathcal{E}(A))\right\rangle_{H S}=\sum_{j=1}^{n} \frac{\left|\operatorname{Tr}\left(F_{j} A\right)\right|^{2}}{\operatorname{Tr}\left(F_{j} \sigma\right)},
$$

which is independent of $\kappa$. By (16), it is straightforward to observe that when $\kappa \geq \kappa_{1 / 2}$, one has $\left\langle A, \Omega_{\sigma}^{\kappa}(A)\right\rangle_{H S} \geq\left\langle A, \Omega_{\sigma}^{\kappa_{1 / 2}}(A)\right\rangle_{H S}$. Thus (34) follows immediately; (35) follows from (34) by taking the supremum over all non-zero $A \in \mathbb{H}_{n}^{0}$ (see (23)).

\section{Proof of Theorem 1}

Setting up: First notice that it is sufficient to prove Theorem 1 for $N=2$. The general case can be straightforwardly proved by mathematical induction on $N$. Next, for the case $N=2$, one direction is trivial: suppose $\rho_{1}$ achieves the maximum in $\eta_{\chi_{\kappa}^{2}}\left(\mathcal{E}_{1}, \sigma_{1}\right)$; let $\rho_{1,2}=\rho_{1} \otimes \sigma_{2}$ and by direct calculation,

$$
\begin{aligned}
\eta_{\chi_{\kappa}^{2}}\left(\mathcal{E}_{1} \otimes \mathcal{E}_{2}, \sigma_{1} \otimes \sigma_{2}\right) & \geq \frac{\chi_{\kappa}^{2}\left(\mathcal{E}_{1} \otimes \mathcal{E}_{2}\left(\rho_{1,2}\right) \| \mathcal{E}_{1} \otimes \mathcal{E}_{2}\left(\sigma_{1} \otimes \sigma_{2}\right)\right)}{\chi_{\kappa}^{2}\left(\rho_{1,2} \| \sigma_{1} \otimes \sigma_{2}\right)} \\
& \stackrel{(18)}{=} \frac{\chi_{\kappa}^{2}\left(\mathcal{E}_{1}\left(\rho_{1}\right) \| \mathcal{E}_{1}\left(\sigma_{1}\right)\right)}{\chi_{\kappa}^{2}\left(\rho_{1} \| \sigma_{1}\right)}=\eta_{\chi_{\kappa}^{2}}\left(\mathcal{E}_{1}, \sigma_{1}\right) .
\end{aligned}
$$

Similarly, by choosing $\rho_{1,2}=\sigma_{1} \otimes \rho_{2}$ where $\rho_{2}$ achieves the maximum in $\eta_{\chi_{\kappa}^{2}}\left(\mathcal{E}_{2}, \sigma_{2}\right)$, we have $\eta_{\chi_{\kappa}^{2}}\left(\mathcal{E}_{1} \otimes \mathcal{E}_{2}, \sigma_{1} \otimes \sigma_{2}\right) \geq \eta_{\chi_{\kappa}^{2}}\left(\mathcal{E}_{2}, \sigma_{2}\right)$. Therefore,

$$
\eta_{\chi_{\kappa}^{2}}\left(\mathcal{E}_{1} \otimes \mathcal{E}_{2}, \sigma_{1} \otimes \sigma_{2}\right) \geq \max \left(\eta_{\chi_{\kappa}^{2}}\left(\mathcal{E}_{1}, \sigma_{1}\right), \eta_{\chi_{\kappa}^{2}}\left(\mathcal{E}_{2}, \sigma_{2}\right)\right)
$$

In the below, we shall prove the other direction, i.e.,

$$
\eta_{\chi_{\kappa}^{2}}\left(\mathcal{E}_{1} \otimes \mathcal{E}_{2}, \sigma_{1} \otimes \sigma_{2}\right) \leq \max \left(\eta_{\chi_{\kappa}^{2}}\left(\mathcal{E}_{1}, \sigma_{1}\right), \eta_{\chi_{\kappa}^{2}}\left(\mathcal{E}_{2}, \sigma_{2}\right)\right)=: \eta_{\max }^{\kappa} .
$$




\section{Notations:}

Since we fix states $\sigma_{m}$ and channels $\mathcal{E}_{m}$ for $m=1,2$ throughout this section, let us denote $\Upsilon_{m}^{\kappa} \equiv$ $\Upsilon_{\mathcal{E}_{m}, \sigma_{m}}^{\kappa}$ for simplicity of notation. By Lemma $10, \Upsilon_{m}^{\kappa}$ has an eigen-basis $\left\{V_{j}^{\kappa, m}\right\}_{j=1}^{n_{m}^{2}}$ associated with eigenvalue $\left\{\theta_{j}^{\kappa, m}\right\}_{j=1}^{n_{m}^{2}}$ with respect to the inner product $\langle\cdot, \cdot\rangle_{\Omega_{\sigma_{m}}^{\kappa}}$ such that

$$
\Upsilon_{m}^{\kappa}\left(V_{j}^{\kappa, m}\right)=\theta_{j}^{\kappa, m} V_{j}^{\kappa, m}, \quad 1 \leq j \leq n_{m}^{2},
$$

where $V_{1}^{\kappa, m}=\sigma_{m}, \theta_{1}^{\kappa, m}=1$ and $V_{j}^{\kappa, m}$ are Hermitian for all $1 \leq j \leq n_{m}^{2}$, since from Lemma 9 $\Upsilon_{m}^{\kappa}$ are both Hermitian-preserving positive semidefinite operators. In addition, we know from Lemma 10 (or say Lemma 9 (iv)) that for both $m=1,2$,

$$
\theta_{j}^{\kappa, m} \leq \eta_{\chi_{\kappa}^{2}}\left(\mathcal{E}_{m}, \sigma_{m}\right) \leq \eta_{\max }^{\kappa}, \quad \text { for any } 2 \leq j \leq n_{m}^{2} .
$$
define

For convenience, let $\sigma=\sigma_{1} \otimes \sigma_{2}$ and $\mathcal{E}=\mathcal{E}_{1} \otimes \mathcal{E}_{2} ;$ let $\Upsilon^{\kappa} \equiv \Upsilon_{\mathcal{E}, \sigma}^{\kappa}$. For any index pair $\mathbf{J}=\left(j_{1}, j_{2}\right)$,

$$
V_{\mathbf{J}}^{\kappa}:=V_{j_{1}}^{\kappa, 1} \otimes V_{j_{2}}^{\kappa, 2}, \quad \theta_{\mathbf{J}}^{\kappa}:=\theta_{j_{1}}^{\kappa, 1} \theta_{j_{2}}^{\kappa, 2} .
$$

Case (I): For $\kappa=\kappa_{1 / 2}$ and any quantum channel. From Lemma 7 part (ii), $\Omega_{\sigma}^{\kappa}$ tensorizes, thus $\Upsilon^{\kappa}=\Upsilon_{1}^{\kappa} \otimes \Upsilon_{2}^{\kappa}$. Next, we can straightforwardly verify that $\left\{V_{\mathbf{J}}^{\kappa}\right\}_{\mathbf{J}}$ (for $\mathbf{J}=\left(j_{1}, j_{2}\right)$ ) is an orthonormal eigenbasis of $\Upsilon^{\kappa}$ with respect to the inner product $\langle\cdot, \cdot \cdot\rangle_{\Omega_{\sigma}^{\kappa}}$, and the associated eigenvalues are $\left\{\theta_{\mathbf{J}}^{\kappa}\right\}_{\mathbf{J}}$. The largest eigenvalue of $\Upsilon^{\kappa}$ on the domain $\operatorname{span}(\sigma)^{\perp} \equiv \mathbb{M}_{n_{1} \times n_{2}}^{0}$ becomes $\max _{\mathbf{J} \neq(1,1)}\left\{\theta_{\mathbf{J}}^{\kappa}\right\}=\eta_{\max }^{\kappa}$. Therefore, by Lemma 10, we have $\eta_{\chi_{\kappa}^{2}}(\mathcal{E}, \sigma)=\max _{\mathbf{J} \neq(1,1)}\left\{\theta_{\mathbf{J}}^{\kappa}\right\}=$ $\max \left(\eta_{\chi_{\kappa}^{2}}\left(\mathcal{E}_{1}, \sigma_{1}\right), \eta_{\chi_{\kappa}^{2}}\left(\mathcal{E}_{2}, \sigma_{2}\right)\right)$. Thus we complete the proof of (37) for the case $\kappa_{1 / 2}$.

Case (II): For $\kappa \geq \kappa_{1 / 2}$ and QC channels. Let us decompose $\mathbf{A} \in \mathbb{H}_{n_{1} \times n_{2}}^{0}$ by $\mathbf{A}=\sum_{\mathbf{J}} c_{\mathbf{J}} V_{\mathbf{J}}^{\kappa}$ where $c_{\mathbf{J}} \in \mathbb{R}$. From the constraint that $\operatorname{Tr}(\mathbf{A})=0$, we know $c_{(1,1)}=0$. Thus, we can rewrite $\mathbf{A}$ by

$$
\mathbf{A}=\sigma_{1} \otimes A_{2}+A_{1} \otimes \sigma_{2}+\widetilde{A}
$$

where

$$
\left\{\begin{array}{l}
A_{2}=\sum_{\mathbf{J}: j_{1}=1,2 \leq j_{2} \leq n_{2}^{2}} c_{\mathbf{J}} V_{j_{2}}^{\kappa, 2} ; \\
A_{1}=\sum_{\mathbf{J}: 2 \leq j_{1} \leq n_{1}^{2}, j_{2}=1} c_{\mathbf{J}} V_{j_{1}}^{\kappa, 1} ; \\
\widetilde{A}=\sum_{\mathbf{J}: j_{1} \neq 1, j_{2} \neq 1} c_{\mathbf{J}} V_{\mathbf{J}} .
\end{array}\right.
$$

To prove (37), by (23), it is equivalent to prove that for all $\mathbf{A} \in \mathbb{H}_{n_{1} \times n_{2}}^{0}$ and $\mathbf{A} \neq 0$, we have

$$
\frac{\left\langle\mathbf{A}, \Upsilon^{\kappa}(\mathbf{A})\right\rangle_{\Omega_{\sigma}^{\kappa}}}{\langle\mathbf{A}, \mathbf{A}\rangle_{\Omega_{\sigma}^{\kappa}}} \leq \eta_{\max }^{\kappa} .
$$

The next lemma shows that it is sufficient to consider $\mathbf{A}$ as $\widetilde{A}$.

Lemma 15. If (40) holds for any $\boldsymbol{A} \in \mathbb{H}_{n_{1}}^{0} \otimes \mathbb{H}_{n_{2}}^{0}$, then (40) holds for any $\boldsymbol{A} \in \mathbb{H}_{n_{1} \times n_{2}}^{0}$.

Notice that $\mathbb{H}_{n_{1}}^{0} \otimes \mathbb{H}_{n_{2}}^{0} \subset \mathbb{H}_{n_{1} \times n_{2}}^{0}$. The proof of this lemma is postponed to the end of this section and let us continue to complete the proof of Theorem 1. It is straightforward to verify that when $\mathcal{E}_{1}$ and $\mathcal{E}_{2}$ are QC channels, $\mathcal{E}=\mathcal{E}_{1} \otimes \mathcal{E}_{2}$ is also a QC channel for the composite system. By Lemma 14, for any $\mathbf{A} \in \mathbb{H}_{n_{1}}^{0} \otimes \mathbb{H}_{n_{2}}^{0}$, we have

$$
\begin{aligned}
\frac{\left\langle\mathbf{A}, \Upsilon^{\kappa}(\mathbf{A})\right\rangle_{\Omega_{\sigma}^{\kappa}}(34)}{\langle\mathbf{A}, \mathbf{A}\rangle_{\Omega_{\sigma}^{\kappa}}} & \leq \frac{\left\langle\mathbf{A}, \Upsilon^{\kappa_{1 / 2}}(\mathbf{A})\right\rangle_{\Omega_{\sigma}^{\kappa_{1 / 2}}}}{\langle\mathbf{A}, \mathbf{A}\rangle_{\Omega_{\sigma}^{\kappa_{1 / 2}}}} \\
& \leq \eta_{\chi_{\kappa_{1 / 2}}^{2}}\left(\mathcal{E}_{1}, \sigma_{1}\right) \eta_{\chi_{\kappa_{1 / 2}}^{2}}\left(\mathcal{E}_{2}, \sigma_{2}\right) \\
& \leq \max \left(\left(\eta_{\chi_{\kappa_{1 / 2}}^{2}}\left(\mathcal{E}_{1}, \sigma_{1}\right)\right)^{2},\left(\eta_{\chi_{\kappa_{1 / 2}}^{2}}\left(\mathcal{E}_{2}, \sigma_{2}\right)\right)^{2}\right) \\
& \stackrel{(28)}{\leq} \max \left(\eta_{\chi_{\kappa}^{2}}\left(\mathcal{E}_{1}, \sigma_{1}\right), \eta_{\chi_{\kappa}^{2}}\left(\mathcal{E}_{2}, \sigma_{2}\right)\right)=\eta_{\max }^{\kappa} .
\end{aligned}
$$


The second inequality comes from the observation that $\Upsilon^{\kappa_{1 / 2}}$ is a positive semidefinite operator on the space $\mathbb{H}_{n_{1}}^{0} \otimes \mathbb{H}_{n_{2}}^{0}$ with eigenvalues $\theta_{\mathbf{J}}^{\kappa_{1 / 2}}$; for $j_{1} \neq 1$ and $j_{2} \neq 1$, recall from previous results that $\theta_{\mathbf{J}}^{\kappa_{1 / 2}}=\theta_{j_{1}}^{\kappa_{1 / 2}, 1} \theta_{j_{2}}^{\kappa_{1 / 2}, 2} \leq \eta_{\chi_{\kappa_{1 / 2}}^{2}}\left(\mathcal{E}_{1}, \sigma_{1}\right) \eta_{\chi_{\kappa_{1 / 2}}^{2}}\left(\mathcal{E}_{2}, \sigma_{2}\right)$. The last equation means (40) holds for all Hermitian $\mathbf{A} \in \mathbb{H}_{n_{1}}^{0} \otimes \mathbb{H}_{n_{2}}^{0}$ and by Lemma 15, (40) holds for all Hermitian $\mathbf{A} \in \mathbb{H}_{n_{1} \times n_{2}}^{0}$. This completes the proof of Theorem 1 .

Proof of Lemma 15. For any Hermitian A in (38), we claim that

$$
\begin{aligned}
\left\langle\mathbf{A}, \Upsilon^{\kappa}(\mathbf{A})\right\rangle_{\Omega_{\sigma}^{\kappa}}=\left\langle\sigma_{1} \otimes A_{2}, \Upsilon^{\kappa}\left(\sigma_{1} \otimes A_{2}\right)\right\rangle_{\Omega_{\sigma}^{\kappa}} & +\left\langle A_{1} \otimes \sigma_{2}, \Upsilon^{\kappa}\left(A_{1} \otimes \sigma_{2}\right)\right\rangle_{\Omega_{\sigma}^{\kappa}} \\
& +\left\langle\widetilde{A}, \Upsilon^{\kappa}(\widetilde{A})\right\rangle_{\Omega_{\sigma}^{\kappa}} .
\end{aligned}
$$

To prove this, we need to show that all cross product terms in the expansion of $\left\langle\mathbf{A}, \Upsilon^{\kappa}(\mathbf{A})\right\rangle_{\Omega_{\sigma}^{\kappa}}$ vanish. For instance, consider any $\mathbf{B} \in \mathbb{M}_{n_{1}} \otimes \mathbb{M}_{n_{2}}$,

$$
\begin{aligned}
& \left\langle\mathbf{B}, \Upsilon^{\kappa}\left(\sigma_{1} \otimes A_{2}\right)\right\rangle_{\Omega_{\sigma}^{\kappa}} \\
\stackrel{(22)}{=} & \left\langle\mathbf{B}, \mathcal{E}^{\dagger} \circ \Omega_{\mathcal{E}(\sigma)}^{\kappa} \circ \mathcal{E}\left(\sigma_{1} \otimes A_{2}\right)\right\rangle_{H S} \\
= & \left\langle\mathbf{B}, \mathcal{E}^{\dagger} \circ \Omega_{\mathcal{E}(\sigma)}^{\kappa}\left(\mathcal{E}_{1}\left(\sigma_{1}\right) \otimes \mathcal{E}_{2}\left(A_{2}\right)\right)\right\rangle_{H S} \\
\stackrel{(18)}{=} & \left\langle\mathbf{B}, \mathcal{E}^{\dagger}\left(\mathbb{I}_{n_{1}} \otimes \Omega_{\mathcal{E}_{2}\left(\sigma_{2}\right)}^{\kappa} \circ \mathcal{E}_{2}\left(A_{2}\right)\right)\right\rangle_{H S} \\
= & \left\langle\mathbf{B}, \mathbb{I}_{n_{1}} \otimes\left(\mathcal{E}_{2}^{\dagger} \circ \Omega_{\mathcal{E}_{2}\left(\sigma_{2}\right)}^{\kappa} \circ \mathcal{E}_{2}\left(A_{2}\right)\right)\right\rangle_{H S} .
\end{aligned}
$$

If $\mathbf{B}=A_{1} \otimes \sigma_{2}$ or $\mathbf{B}=\widetilde{A}$, by plugging the expression of $A_{1}$ or $\widetilde{A}$ into the last equation and after expanding all terms, it is straightforward to verify that $\left\langle\mathbf{B}, \Upsilon^{\kappa}\left(\sigma_{1} \otimes A_{2}\right)\right\rangle_{\Omega_{\sigma}^{\kappa}}=0$ for both choices of B. We can apply similar arguments to $\left\langle\mathbf{B}, \Upsilon^{\kappa}\left(A_{1} \otimes \sigma_{2}\right)\right\rangle_{\Omega_{\sigma}^{\kappa}}$ for $\mathbf{B}=\sigma_{1} \otimes A_{2}$ or $\mathbf{B}=\widetilde{A}$. Similarly, we have (or let $\mathcal{E}=\mathcal{I}_{n_{1}} \otimes \mathcal{I}_{n_{2}}$ in (41))

$$
\begin{aligned}
\langle\mathbf{A}, \mathbf{A}\rangle_{\Omega_{\sigma}^{\kappa}} & =\left\langle\sigma_{1} \otimes A_{2}, \sigma_{1} \otimes A_{2}\right\rangle_{\Omega_{\sigma}^{\kappa}}+\left\langle A_{1} \otimes \sigma_{2}, A_{1} \otimes \sigma_{2}\right\rangle_{\Omega_{\sigma}^{\kappa}}+\langle\widetilde{A}, \widetilde{A}\rangle_{\Omega_{\sigma}^{\kappa}} \\
& \stackrel{(18)}{=}\left\langle A_{2}, A_{2}\right\rangle_{\Omega_{\sigma_{2}}}+\left\langle A_{1}, A_{1}\right\rangle_{\Omega_{\sigma_{1}}}+\langle\widetilde{A}, \widetilde{A}\rangle_{\Omega_{\sigma}^{\kappa}} .
\end{aligned}
$$

Let us simplify the term on the right hand side of (41). For instance,

$$
\begin{aligned}
& \left\langle\sigma_{1} \otimes A_{2}, \Upsilon^{\kappa}\left(\sigma_{1} \otimes A_{2}\right)\right\rangle_{\Omega_{\sigma}^{\kappa}} \\
= & \left\langle\mathcal{E}\left(\sigma_{1} \otimes A_{2}\right), \Omega_{\mathcal{E}(\sigma)}^{\kappa} \circ \mathcal{E}\left(\sigma_{1} \otimes A_{2}\right)\right\rangle_{H S} \\
\stackrel{(18)}{=} & \left\langle\mathcal{E}_{1}\left(\sigma_{1}\right) \otimes \mathcal{E}_{2}\left(A_{2}\right), \mathbb{I}_{n_{1}} \otimes \Omega_{\mathcal{E}_{2}\left(\sigma_{2}\right)}^{\kappa} \circ \mathcal{E}_{2}\left(A_{2}\right)\right\rangle_{H S} \\
= & \left\langle\mathcal{E}_{2}\left(A_{2}\right), \Omega_{\mathcal{E}_{2}\left(\sigma_{2}\right)}^{\kappa} \circ \mathcal{E}_{2}\left(A_{2}\right)\right\rangle_{H S} \\
= & \left\langle A_{2}, \Upsilon_{2}^{\kappa}\left(A_{2}\right)\right\rangle_{\Omega_{\sigma_{2}}^{\kappa}} \\
\leq & \eta_{\chi_{\kappa}^{2}}\left(\mathcal{E}_{2}, \sigma_{2}\right)\left\langle A_{2}, A_{2}\right\rangle_{\Omega_{\sigma_{2}}^{\kappa}} \\
\leq & \eta_{\max }^{\kappa}\left\langle A_{2}, A_{2}\right\rangle_{\Omega_{\sigma_{2}}^{\kappa}} .
\end{aligned}
$$

Similarly,

$$
\left\langle A_{1} \otimes \sigma_{2}, \Upsilon^{\kappa}\left(A_{1} \otimes \sigma_{2}\right)\right\rangle_{\Omega_{\sigma}^{\kappa}} \leq \eta_{\max }^{\kappa}\left\langle A_{1}, A_{1}\right\rangle_{\Omega_{\sigma_{1}}^{\kappa}}
$$

Therefore, we have

$$
\left\langle\mathbf{A}, \Upsilon^{\kappa}(\mathbf{A})\right\rangle_{\Omega_{\sigma}^{\kappa}} \leq \eta_{\max }^{\kappa}\left(\left\langle A_{2}, A_{2}\right\rangle_{\Omega_{\sigma_{2}}^{\kappa}}+\left\langle A_{1}, A_{1}\right\rangle_{\Omega_{\sigma_{1}}^{\kappa}}\right)+\left\langle\widetilde{A}, \Upsilon^{\kappa}(\widetilde{A})\right\rangle_{\Omega_{\sigma}^{\kappa}} .
$$


By comparing (42) and (43), to prove (40), it is sufficient to show

$$
\left\langle\widetilde{A}, \Upsilon^{\kappa}(\widetilde{A})\right\rangle_{\Omega_{\sigma}^{\kappa}} \leq \eta_{\max }^{\kappa}\langle\widetilde{A}, \widetilde{A}\rangle_{\Omega_{\sigma}^{\kappa}}
$$

Thus we complete the proof of Lemma 15.

\section{Connection to the quantum maximal correlation}

The SDPI constant for the classical $\chi^{2}$ divergence is closely connected to the classical maximal correlation (see e.g., [18, Theorem III.2]). In the proposition below, we provide a quantum analog of this relation when $\kappa=\kappa_{1 / 2}$.

To begin with, we need to define the quantum maximal correlation. This concept was previously proposed and studied in [2]. Since there is a whole family of quantum $\chi_{\kappa}^{2}$ divergences, it is natural to imagine that there could also exist a whole family of quantum maximal correlations, as a straightforward generalization of [2].

Definition 16 ( $\kappa$-quantum maximal correlation). Consider any fixed $\kappa \in \mathcal{K}$ and Hilbert spaces $\mathcal{H}_{1}$ and $\mathcal{H}_{2}$ with dimensions $n_{1}$ and $n_{2}$ respectively. For any bipartite quantum state $\rho_{1,2}$ on the composite system $\mathcal{H}_{1} \otimes \mathcal{H}_{2}$, denote the reduced density matrices by $\rho_{1}$ and $\rho_{2}$ respectively (i.e., $\left.\operatorname{Tr}_{2}\left(\rho_{1,2}\right)=\rho_{1}, \operatorname{Tr}_{1}\left(\rho_{1,2}\right)=\rho_{2}\right)$. Define the $\kappa$-quantum maximal correlation $\mu_{\kappa}\left(\rho_{1,2}\right)$ by

$$
\mu_{\kappa}\left(\rho_{1,2}\right):=\max \left|\operatorname{Tr}\left(\rho_{1,2} F \otimes G^{\dagger}\right)\right|
$$

where the maximum is taken over all $F \in \mathbb{M}_{n_{1}}, G \in \mathbb{M}_{n_{2}}$ such that

$$
\left\langle\mathbb{I}_{n_{1}}, F\right\rangle_{\mho_{\rho_{1}}^{\kappa}}=\left\langle\mathbb{I}_{n_{2}}, G\right\rangle_{\mho_{\rho_{2}}^{\kappa}}=0, \quad\|F\|_{\mho_{\rho_{1}}^{\kappa}}=\|G\|_{\mho_{\rho_{2}}^{\kappa}}=1
$$

Technically, when $\rho_{1}$ is not a full-rank density matrix, the notation $\langle\cdot, \cdot\rangle_{\mho_{\rho_{1}}^{\kappa}}$ should be understood as a sesquilinear form, as we explained at the beginning of $\S 2$ and the operator $\mho_{\rho_{1}}^{\kappa}$ is still welldefined on the support of $\rho_{1}$ via (20). By Lemma 8, we easily verify that $\left\langle\mathbb{I}_{n_{1}}, F\right\rangle_{\mho_{\rho_{1}}^{\kappa}} \equiv \operatorname{Tr}\left(\rho_{1} F\right)$ and $\left\langle\mathbb{I}_{n_{2}}, G\right\rangle_{\mho_{\rho_{2}}} \equiv \operatorname{Tr}\left(\rho_{2} G\right)$. When $\kappa(x)=1$ is a constant function, we recover the quantum maximal correlation defined in [2]; in this case, $\mho_{\sigma}^{\kappa(x)=1}=L_{\sigma}$; however, notice that this choice of $\kappa$ is not included in the set $\mathcal{K}$ and the corresponding operator $\mho_{\sigma}^{\kappa(x)=1}$ is not Hermitian-preserving.

Lemma 17 (Invariance of the $\kappa$-quantum maximal correlation under local isometries). Suppose $U: \mathcal{H}_{1} \rightarrow \widetilde{\mathcal{H}}_{1}$ and $V: \mathcal{H}_{2} \rightarrow \widetilde{\mathcal{H}}_{2}$ are two isometries (i.e., $U^{\dagger} U=\mathbb{I}_{\operatorname{dim}\left(\mathcal{H}_{1}\right)}$ and $V^{\dagger} V=\mathbb{I}_{\operatorname{dim}\left(\mathcal{H}_{2}\right)}$ ), where $\operatorname{dim}\left(\mathcal{H}_{1}\right) \leq \operatorname{dim}\left(\widetilde{\mathcal{H}}_{1}\right)$ and $\operatorname{dim}\left(\mathcal{H}_{2}\right) \leq \operatorname{dim}\left(\widetilde{\mathcal{H}}_{2}\right)$. For any bipartite quantum state $\rho_{1,2}$ on $\mathcal{H}_{1} \otimes \mathcal{H}_{2}$, define $\widetilde{\rho}_{1,2}:=(U \otimes V) \rho(U \otimes V)^{\dagger}$. We have

$$
\mu_{\kappa}\left(\rho_{1,2}\right)=\mu_{\kappa}\left(\widetilde{\rho}_{1,2}\right)
$$

Proof. By definition,

$$
\begin{aligned}
\mu_{\kappa}\left(\widetilde{\rho}_{1,2}\right) & =\max _{\widetilde{F}, \widetilde{G}}\left|\operatorname{Tr}\left(\widetilde{\rho}_{1,2} \widetilde{F} \otimes \widetilde{G}^{\dagger}\right)\right| \\
& =\max _{\widetilde{F}, \widetilde{G}}\left|\operatorname{Tr}\left(\rho_{1,2}\left(U^{\dagger} \widetilde{F} U\right) \otimes\left(V^{\dagger} \widetilde{G}^{\dagger} V\right)\right)\right|=\max _{\widetilde{F}, \widetilde{G}}\left|\operatorname{Tr}\left(\rho_{1,2} F \otimes G^{\dagger}\right)\right|,
\end{aligned}
$$

where we define $F:=U^{\dagger} \widetilde{F} U$ and $G:=V^{\dagger} \widetilde{G} V$. Denote the reduced density matrices of $\rho_{1,2}$ as $\rho_{1}$ and $\rho_{2}$ respectively. Then the reduced density matrices of $\widetilde{\rho}_{1,2}$ are given by $\widetilde{\rho}_{1}:=U \rho_{1} U^{\dagger}$ and $\widetilde{\rho}_{2}:=V \rho_{2} V^{\dagger}$ respectively. From (46), the condition in the maximization is given by

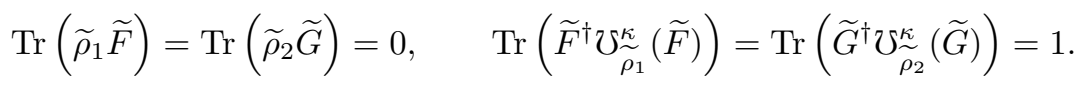


By $(20)$, it could be readily shown that $\mho_{\rho_{1}}^{\kappa}(\cdot)=\left(U \# U^{\dagger}\right) \circ \mho_{\rho_{1}}^{\kappa} \circ\left(U^{\dagger} \# U\right)$ and similarly for $\mho_{\rho_{2}}^{\kappa}(\cdot)$. As a remark, in this case, $\widetilde{\rho}_{1}$ and $\widetilde{\rho}_{2}$ might not be strictly positive, then the decomposition in ${ }^{\rho_{2}}(20)$ only considers eigenstates with respect to non-zero eigenvalues (i.e., $\mho_{\tilde{\rho}_{1}}^{\kappa}$ is only defined on the support of $\left.\widetilde{\rho}_{1}\right)$. Then, with direct calculation, one could verify that the above four conditions are equivalent to

$$
\operatorname{Tr}\left(\rho_{1} F\right)=\operatorname{Tr}\left(\rho_{2} G\right)=0, \quad \operatorname{Tr}\left(F^{\dagger} \mho_{\rho_{1}}^{\kappa}(F)\right)=\operatorname{Tr}\left(G^{\dagger} \mho_{\rho_{2}}^{\kappa}(G)\right)=1 .
$$

Therefore, we know $\mu_{\kappa}\left(\widetilde{\rho}_{1,2}\right) \leq \mu_{\kappa}\left(\rho_{1,2}\right)$. Since $\widetilde{F}$ is a linear operator on a higher-dimensional Hilbert space $\widetilde{\mathcal{H}}_{1}$ than $F$ on $\mathcal{H}_{1}$, for any such $F$, there exists $\widetilde{F}$ such that $U^{\dagger} \widetilde{F} U=F$ (similarly for $G)$; therefore the equality can be achieved and $\mu_{\kappa}\left(\widetilde{\rho}_{1,2}\right)=\mu_{\kappa}\left(\rho_{1,2}\right)$.

Theorem 18. For a Hilbert space $\mathcal{H}$ with dimension $n$, suppose $\sigma \in \mathfrak{D}_{n}^{+}$and $\mathcal{E}$ is any quantum channel on $\mathcal{H}$ such that the quantum state $\mathcal{E}(\sigma) \in \mathfrak{D}_{n}^{+}$. Thus, $\sigma$ has an eigenvalue decomposition $\sigma=\sum_{j=1}^{n} s_{j}\left|s_{j}\right\rangle\left\langle s_{j}\right|$. For the choice $\kappa=\kappa_{1 / 2}$,

$$
\sqrt{\eta_{\chi_{\kappa_{1 / 2}}^{2}}(\mathcal{E}, \sigma)}=\mu_{\kappa_{1 / 2}}\left(\rho_{1,2}\right)
$$

where the bipartite quantum state $\rho_{1,2}:=\left(\mathbb{I}_{n} \otimes \mathcal{E}\right)(|\psi\rangle\langle\psi|)$ and the wave function $|\psi\rangle$ is any purification of $\sigma$ on the system $\mathcal{H} \otimes \mathcal{H}$.

Recall that a pure state $|\psi\rangle$ on $\mathcal{H} \otimes \mathcal{H}$ is a purification of $\sigma$ if $\operatorname{Tr}_{1}(|\psi\rangle\langle\psi|)=\sigma$ (see [23, Chap. 5]). The canonical choice of the purification $|\psi\rangle$ of $\sigma$ is

$$
\left|\psi_{\mathrm{c}}\right\rangle:=\sum_{j=1}^{n} \sqrt{s_{j}}\left|s_{j}, s_{j}\right\rangle .
$$

Proof. In the first step, we prove it for the choice $|\psi\rangle=\left|\psi_{c}\right\rangle$; in the second step, we extend the result to the general purification.

Step (I). By Lemma 11, we have

$$
\sqrt{\eta_{\chi_{\kappa}^{2}}(\mathcal{E}, \sigma)}=\max _{F, G}\left|\left\langle\mathcal{E} \circ \Gamma_{\sigma}(F), \widetilde{G}\right\rangle_{H S}\right|=\max _{F, G}\left|\left\langle\widetilde{G}, \mathcal{E} \circ \Gamma_{\sigma}(F)\right\rangle_{H S}\right|,
$$

where $\widetilde{G}:=\left(\Gamma_{\mathcal{E}(\sigma)}\right)^{-1} \circ \mho_{\mathcal{E}(\sigma)}^{\kappa}(G)$. Let us decompose $\mathcal{E} \circ \Gamma_{\sigma}(F)$ based on the eigenstates of $\sigma$,

$$
\mathcal{E} \circ \Gamma_{\sigma}(F)=\sum_{j, m=1}^{n} \sqrt{s_{m} s_{j}}\left\langle s_{m}|F| s_{j}\right\rangle \mathcal{E}\left(\left|s_{m}\right\rangle\left\langle s_{j}\right|\right) .
$$

Hence,

$$
\begin{aligned}
\sqrt{\eta_{\chi_{\kappa}^{2}}(\mathcal{E}, \sigma)} & =\max _{F, G}\left|\sum_{j, m=1}^{n} \sqrt{s_{m} s_{j}}\left\langle s_{m}|F| s_{j}\right\rangle\left\langle\widetilde{G}, \mathcal{E}\left(\left|s_{m}\right\rangle\left\langle s_{j}\right|\right)\right\rangle_{H S}\right| \\
& =\max _{F, G}\left|\sum_{j, m=1}^{n} \sqrt{s_{m} s_{j}}\left\langle s_{j}|\widetilde{F}| s_{m}\right\rangle\left\langle\widetilde{G}, \mathcal{E}\left(\left|s_{m}\right\rangle\left\langle s_{j}\right|\right)\right\rangle_{H S}\right| \\
& =\max _{F, G}\left|\operatorname{Tr}\left(\rho_{1,2} \widetilde{F} \otimes \widetilde{G}^{\dagger}\right)\right|,
\end{aligned}
$$

where $\widetilde{F}=F^{T}$ and the superscript $T$ means transpose with respect to the eigenstates of $\sigma$, i.e., $\left\langle s_{j}|\widetilde{F}| s_{m}\right\rangle:=\left\langle s_{m}|F| s_{j}\right\rangle$ for all $1 \leq j, m \leq n$. The last equality above can be verified directly by $\rho_{1,2}=\left(\mathcal{I}_{n} \otimes \mathcal{E}\right)\left(\left|\psi_{c}\right\rangle\left\langle\psi_{c}\right|\right)$.

Notice that from Lemma 11, the maximum is taken over all $F, G$ given in (27). Hence, to prove Theorem 18, it remains to verify that conditions (27) for $F$ and $G$ are equivalent to conditions (46) for $\widetilde{F}$ and $\widetilde{G}$. More specifically, we need to verify the following four relations. 
(i) $\left\langle\mathbb{I}_{n}, F\right\rangle_{\mho_{\sigma}^{\kappa}}=\left\langle\mathbb{I}_{n}, \widetilde{F}\right\rangle_{\mho_{\sigma}^{\kappa}}$. Note that

$$
\left\langle\mathbb{I}_{n}, F\right\rangle_{\mho_{\sigma}^{\kappa}}=\operatorname{Tr}(\sigma F)=\sum_{j} s_{j}\left\langle s_{j}|F| s_{j}\right\rangle=\sum_{j} s_{j}\left\langle s_{j}|\widetilde{F}| s_{j}\right\rangle=\operatorname{Tr}(\sigma \widetilde{F})=\left\langle\mathbb{I}_{n}, \widetilde{F}\right\rangle_{\mho_{\sigma}^{\kappa}} .
$$

(ii) $\|F\|_{\mho_{\sigma}^{\kappa}}=\|\widetilde{F}\|_{\mho_{\sigma}^{\kappa}}$. Note that

$$
\begin{aligned}
\|F\|_{\mho_{\sigma}^{\kappa}}^{2} & =\operatorname{Tr}\left(F^{\dagger} \mho_{\sigma}^{\kappa}(F)\right) \stackrel{(20)}{=} \sum_{j, m} s_{j} \kappa\left(\frac{s_{j}}{s_{m}}\right)\left|\left\langle s_{j}|F| s_{m}\right\rangle\right|^{2} \\
& =\sum_{j, m} s_{j} \kappa\left(\frac{s_{j}}{s_{m}}\right)\left|\left\langle s_{m}|\widetilde{F}| s_{j}\right\rangle\right|^{2} \stackrel{(7)}{=} \sum_{j, m} s_{m} \kappa\left(\frac{s_{m}}{s_{j}}\right)\left|\left\langle s_{m}|\widetilde{F}| s_{j}\right\rangle\right|^{2} \\
& =\operatorname{Tr}\left((\widetilde{F})^{\dagger} \mho_{\sigma}^{\kappa}(\widetilde{F})\right)=\|\widetilde{F}\|_{\mho_{\sigma}^{\kappa}}^{2} .
\end{aligned}
$$

(iii) $\left\langle\mathbb{I}_{n}, G\right\rangle_{\mho_{\mathcal{E}(\sigma)}^{\kappa}}=\left\langle\mathbb{I}_{n}, \widetilde{G}\right\rangle_{\mho_{\mathcal{E}(\sigma)}^{\kappa}}$. Note that

$$
\left\langle\mathbb{I}_{n}, \widetilde{G}\right\rangle_{\mho_{\mathcal{E}(\sigma)}^{\kappa}}=\left\langle\mathcal{E}(\sigma), \Gamma_{\mathcal{E}(\sigma)}^{-1} \circ \mho_{\mathcal{E}(\sigma)}^{\kappa}(G)\right\rangle_{H S}=\langle\mathcal{E}(\sigma), G\rangle_{H S}=\left\langle\mathbb{I}_{n}, G\right\rangle_{\mho_{\mathcal{E}(\sigma)}^{\kappa}} .
$$

(iv) $\|G\|_{\mho_{\mathcal{E}(\sigma)}^{\kappa}}=\|\widetilde{G}\|_{\mho_{\mathcal{E}(\sigma)}^{\kappa}}$. Note that

$$
\begin{aligned}
\|\widetilde{G}\|_{\mho_{\mathcal{E}(\sigma)}^{\kappa}}^{2} & =\left\langle\widetilde{G}, \mho_{\mathcal{E}(\sigma)}^{\kappa}(\widetilde{G})\right\rangle_{H S}=\left\langle\Gamma_{\mathcal{E}(\sigma)}^{-1} \circ \mho_{\mathcal{E}(\sigma)}^{\kappa}(G), \mho_{\mathcal{E}(\sigma)}^{\kappa} \circ \Gamma_{\mathcal{E}(\sigma)}^{-1} \circ \mho_{\mathcal{E}(\sigma)}^{\kappa}(G)\right\rangle_{H S} \\
& =\left\langle G,\left(\Gamma_{\mathcal{E}(\sigma)}^{-1} \circ \mho_{\mathcal{E}(\sigma)}^{\kappa}\right)^{2}(G)\right\rangle_{\mho_{\mathcal{E}(\sigma)}^{\kappa}} .
\end{aligned}
$$

When $\kappa=\kappa_{1 / 2}, \Gamma_{\mathcal{E}(\sigma)}^{-1} \circ \mho_{\mathcal{E}(\sigma)}^{\kappa}=\mathcal{I}_{n}$. Thus the relation holds for this special choice of $\kappa$ and this is the only place we employ this assumption.

Step (II): We then extend the result from the canonical purification $\left|\psi_{c}\right\rangle$ to any purification $|\psi\rangle$ on the bipartite quantum system $\mathcal{H} \otimes \mathcal{H}$. By [23, Theorem 5.1.1], there exists a unitary (thus also isometry) $U: \mathcal{H} \rightarrow \mathcal{H}$ such that $|\psi\rangle=U \otimes \mathbb{I}_{n}\left|\psi_{c}\right\rangle$. Hence, $\left(\mathcal{I}_{n} \otimes \mathcal{E}\right)(|\psi\rangle\langle\psi|)=(U \otimes$ $\left.\mathbb{I}_{n}\right)\left(\left(\mathcal{I}_{n} \otimes \mathcal{E}\right)\left(\left|\psi_{c}\right\rangle\left\langle\psi_{c}\right|\right)\right)\left(U \otimes \mathbb{I}_{n}\right)^{\dagger}$. By Lemma 17 , the conclusion follows immediately.

\section{SDPI constants for special qubit channels}

In this section, we will illustrate the dependence of SDPI constants on the reference state $\sigma$ and the weight function $\kappa$, for several special qubit channels. The dependence on $\sigma$ is one major difference between the quantum SDPI framework and the quantum contraction coefficient approach. The dependence on $\kappa$ is one major difference between the quantum SDPI framework and its classical version: all quantum $\chi_{\kappa}^{2}$ divergences coincide for classical states $\rho$ and $\sigma$ (i.e., $\rho$ and $\sigma$ commute) and simply reduce to the classical $\chi^{2}$ divergence; in particular, classical $\chi^{2}$ divergence, as well as the associated classical SDPI constant, does not depend on $\kappa$; however, the SDPI constant for quantum $\chi_{\kappa}^{2}$ divergences might fluctuate significantly between approximately 0 and 1 for various $\kappa$, in a special example that we provide below.

Three Pauli matrices are denoted by $\sigma_{X}, \sigma_{Y}, \sigma_{Z}$. Without loss of generality, assume $\sigma=$ $\frac{1}{2}\left(\mathbb{I}_{2}+s \sigma_{Z}\right)=\left[\begin{array}{cc}(1+s) / 2 & 0 \\ 0 & (1-s) / 2\end{array}\right]$ with $s \in[0,1)$, because one can always choose the eigenbasis of $\sigma$ as the computational basis; of course, the matrix representation of the quantum channel is changed, by choosing such a specific computational basis. 


\subsection{QC channel}

By the expression of QC channel (32) and by (36), we have for any $A \in \mathbb{H}_{2}^{0}$ that

$$
\left\langle\mathcal{E}(A), \Omega_{\mathcal{E}(\sigma)}^{\kappa}(\mathcal{E}(A))\right\rangle_{H S}=\sum_{j=1}^{2} \frac{\left|\operatorname{Tr}\left(F_{j} A\right)\right|^{2}}{\operatorname{Tr}\left(F_{j} \sigma\right)}=\left|\operatorname{Tr}\left(F_{1} A\right)\right|^{2}\left(\frac{1}{\operatorname{Tr}\left(F_{1} \sigma\right)}+\frac{1}{1-\operatorname{Tr}\left(F_{1} \sigma\right)}\right) .
$$

The second equality comes from the fact that $F_{2}=\mathbb{I}_{2}-F_{1}$ and $\operatorname{Tr}(A)=0$. Let us decompose $A=a_{x} \sigma_{X}+a_{y} \sigma_{Y}+a_{z} \sigma_{Z}$ and $F_{1}=f_{0} \mathbb{I}_{2}+f_{x} \sigma_{X}+f_{y} \sigma_{Y}+f_{z} \sigma_{Z}$; notice that all coefficients for $A$ and $F_{1}$ are real numbers. Next, rewrite the above equation by

$$
\left\langle\mathcal{E}(A), \Omega_{\mathcal{E}(\sigma)}^{\kappa}(\mathcal{E}(A))\right\rangle_{H S}=\frac{4}{\operatorname{Tr}\left(F_{1} \sigma\right)\left(1-\operatorname{Tr}\left(F_{1} \sigma\right)\right)}\left(f_{x} a_{x}+f_{y} a_{y}+f_{z} a_{z}\right)^{2} .
$$

From (16), we also have

$$
\left\langle A, \Omega_{\sigma}^{\kappa}(A)\right\rangle_{H S}=c_{s}\left(a_{x}^{2}+a_{y}^{2}\right)+\frac{4}{1-s^{2}} a_{z}^{2}
$$

where

$$
c_{s}:=\kappa\left(\frac{1+s}{1-s}\right) \frac{2}{1-s}+\kappa\left(\frac{1-s}{1+s}\right) \frac{2}{1+s} \stackrel{(7)}{=} \frac{4}{1-s} \kappa\left(\frac{1+s}{1-s}\right) \stackrel{(7)}{=} \frac{4}{1+s} \kappa\left(\frac{1-s}{1+s}\right) .
$$

By the Cauchy-Schwarz inequality and the fact that $1-s^{2}>0$ and $c_{s}>0$, we have

$$
\left(f_{x} a_{x}+f_{y} a_{y}+f_{z} a_{z}\right)^{2} \leq\left(\frac{f_{x}^{2}}{c_{s}}+\frac{f_{y}^{2}}{c_{s}}+\frac{f_{z}^{2}}{4 /\left(1-s^{2}\right)}\right)\left(c_{s}\left(a_{x}^{2}+a_{y}^{2}\right)+\frac{4}{1-s^{2}} a_{z}^{2}\right) .
$$

Hence, we know that

$$
\begin{aligned}
\eta_{\chi_{\kappa}^{2}}(\mathcal{E}, \sigma) & =\frac{4}{\operatorname{Tr}\left(F_{1} \sigma\right)\left(1-\operatorname{Tr}\left(F_{1} \sigma\right)\right)}\left(\frac{f_{x}^{2}}{c_{s}}+\frac{f_{y}^{2}}{c_{s}}+\frac{f_{z}^{2}}{4 /\left(1-s^{2}\right)}\right) \\
& =\frac{4}{\left(f_{0}+s f_{z}\right)\left(1-f_{0}-s f_{z}\right)}\left(\frac{f_{x}^{2}}{c_{s}}+\frac{f_{y}^{2}}{c_{s}}+\frac{f_{z}^{2}}{4 /\left(1-s^{2}\right)}\right) .
\end{aligned}
$$

As we can observe, the SDPI constant $\eta_{\chi_{\kappa}^{2}}(\mathcal{E}, \sigma)$ depends on $\kappa$ and the parameter $s$ in a complicated way; however, it does not depend on the choice of pure states in the post-measurement preparation in (32). In the following, let us consider a few special choices of the POVM $\left\{F_{1}, \mathbb{I}_{2}-F_{1}\right\}$.

(High dependence on $\sigma$, for the quantum implementation of BSC)

If $F_{1}=\left[\begin{array}{cr}1-\epsilon & 0 \\ 0 & \epsilon\end{array}\right]$ (thus $F_{2}=\left[\begin{array}{cc}\epsilon & 0 \\ 0 & 1-\epsilon\end{array}\right]$ ) with $\epsilon \in[0,1]$, then the channel $\mathcal{E}$ is exactly a quantum implementation of the binary symmetric channel with crossover probability $\epsilon$ (or BSC $(\epsilon)$ in short). Easily, we know $f_{0}=\frac{1}{2}, f_{z}=\frac{1-2 \epsilon}{2}, f_{x}=f_{y}=0$ and thus the SDPI constant can be simplified as

$$
\eta_{\chi_{\kappa}^{2}}(\mathcal{E}, \sigma)=(1-2 \epsilon)^{2} \frac{1-s^{2}}{1-(1-2 \epsilon)^{2} s^{2}} \leq(1-2 \epsilon)^{2}
$$

Notice that the SDPI constant in this case is independent of the choice of $\kappa$; the upper bound comes from the fact that $\epsilon \in[0,1]$. When we further let $s=0$, i.e., the reference state $\sigma$ has the distribution $\operatorname{Bern}\left(\frac{1}{2}\right)$, the SDPI constant achieves the upper bound $(1-2 \epsilon)^{2}$, which recovers $[18$, Example III.1]. In Figure 1, we show $\eta_{\chi_{\kappa}^{2}}(\mathcal{E}, \sigma)$ with respect to the parameter $s$ in $\sigma$, for fixed $\epsilon=0.05$; the high dependence of $\eta_{\chi_{\kappa}^{2}}$ on $s$ (i.e., on $\sigma$ ) can be clearly seen, for this particular case. 


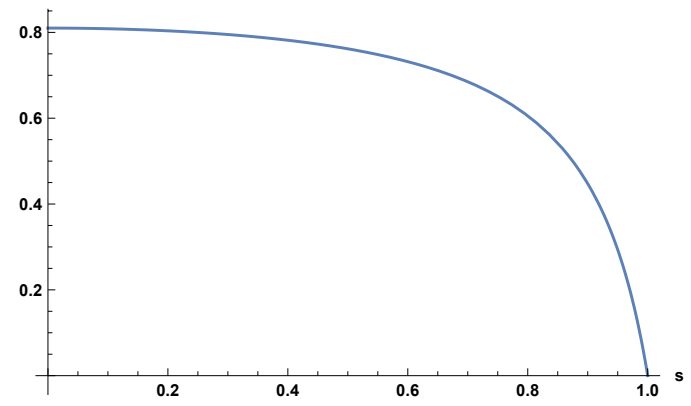

Figure 1: The SDPI constant $\eta_{\chi_{\kappa}^{2}}(\mathcal{E}, \sigma)$ with respect to $s$, for $\operatorname{BSC}(0.05)$.

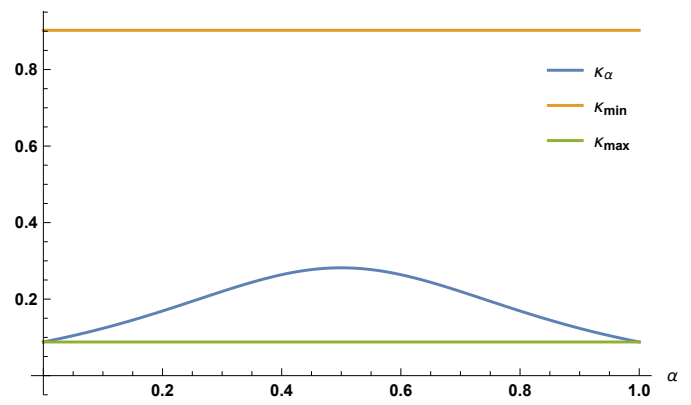

(a) $\eta_{\chi_{\kappa}^{2}}(\mathcal{E}, \sigma)$ for the family of $\kappa_{\alpha}$ in $(13)$.

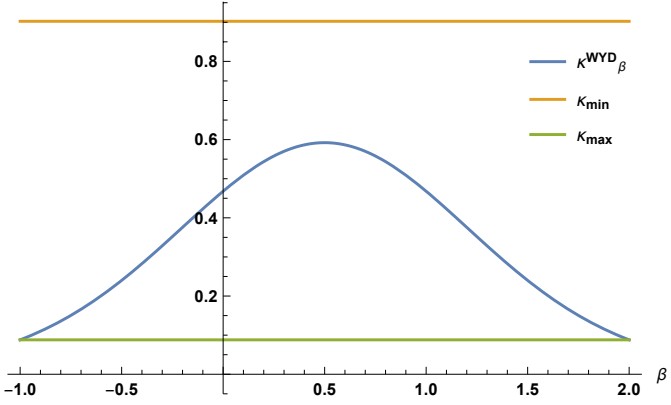

(b) $\eta_{\chi_{\kappa}^{2}}(\mathcal{E}, \sigma)$ for the family of $\kappa_{\beta}^{W Y D}$ in (14).

Figure 2: The SDPI constant $\eta_{\chi_{\kappa}^{2}}(\mathcal{E}, \sigma)$ with respect to various choices of $\kappa$ for the QC channel $\mathcal{E}$ with $F_{1}=\frac{1}{2}\left(\mathbb{I}_{2}+\xi \sigma_{X}\right), \xi=0.95$, and $s=0.95$.

(High dependence on $\kappa$ ).

If $F_{1}=\frac{1}{2}\left(\mathbb{I}_{2}+\xi \sigma_{X}\right)$ with $\xi \in[-1,1]$, then $f_{0}=\frac{1}{2}, f_{x}=\frac{\xi}{2}$ and $f_{y}=f_{z}=0$. Hence,

$$
\eta_{\chi_{\kappa}^{2}}(\mathcal{E}, \sigma)=\frac{4 \xi^{2}}{c_{s}} \stackrel{(50)}{=} \frac{(1+s) \xi^{2}}{\kappa\left(\frac{1-s}{1+s}\right)} \leq \xi^{2} .
$$

The inequality comes from the fact for any $\kappa \in \mathcal{K}$, we have $\kappa(x) \geq \kappa_{\min }(x) \equiv \frac{2}{1+x}$ (see [8, Eq. $(11)])$. As one could observe, even for this simple example, the dependence of $\eta_{\chi_{\kappa}^{2}}(\mathcal{E}, \sigma)$ on $s$ and $\kappa$ is nonlinear and slightly complicated. Similarly, by the fact that for any $\kappa \in \mathcal{K}$, we have $\kappa(x) \leq \kappa_{\max }(x) \equiv \frac{1+x}{2 x}$ (see [8, Eq. (11)]), one could immediately show that

$$
\eta_{\chi_{\kappa}^{2}}(\mathcal{E}, \sigma) \geq\left(1-s^{2}\right) \xi^{2}
$$

Notice that both upper and lower bounds in the above can be achieved for some $\kappa \in \mathcal{K}$. When $s \approx 1$ and $\xi \approx 1$, the largest value of $\eta_{\chi_{\kappa}^{2}}(\mathcal{E}, \sigma)$ is approximately 1 , while the smallest value is approximately 0 , which illustrates the high dependence of $\eta_{\chi_{\kappa}^{2}}(\mathcal{E}, \sigma)$ on the choice of $\kappa$, for this extreme case. In Figure 2, we visualize the SDPI constant $\eta_{\chi_{\kappa}^{2}}(\mathcal{E}, \sigma)$ with respect to various choices of $\kappa$, for $\xi=s=0.95$; the high dependence of $\eta_{\chi_{\kappa}^{2}}(\mathcal{E}, \sigma)$ on $\kappa$ can be clearly observed.

\subsection{Depolarizing channel}

The depolarizing channel on a qubit has the following form

$$
\mathcal{E}(\rho)=\epsilon \rho+(1-\epsilon) \operatorname{Tr}(\rho) \frac{\mathbb{I}_{2}}{2},
$$

for $\epsilon \in[0,1]$. It refers to a physical process in which for a given input state $\rho$, one prepares $\rho$ with probability $\epsilon$ and prepares the maximal mixed state $\frac{\mathbb{I}_{2}}{2}$ with probability $1-\epsilon$. Easily, we know 
that $\mathcal{E}(\sigma)=\frac{\mathbb{I}_{2}}{2}+\frac{s \epsilon}{2} \sigma_{Z}$, and $\mathcal{E}(A)=\epsilon A$ for any $A \in \mathbb{H}_{2}^{0}$. Hence,

$$
\begin{aligned}
\frac{\left\langle\mathcal{E}(A), \Omega_{\mathcal{E}(\sigma)}^{\kappa}(\mathcal{E}(A))\right\rangle_{H S}}{\left\langle A, \Omega_{\sigma}^{\kappa}(A)\right\rangle_{H S}} & =\epsilon^{2} \frac{\left\langle A, \Omega_{\mathcal{E}(\sigma)}^{\kappa}(A)\right\rangle_{H S} \stackrel{(15)}{=} \epsilon^{2} \frac{\frac{4}{1-s^{2} \epsilon^{2}} a_{z}^{2}+c_{s \epsilon}\left(a_{x}^{2}+a_{y}^{2}\right)}{\frac{4}{1-s^{2}} a_{z}^{2}+c_{s}\left(a_{x}^{2}+a_{y}^{2}\right)}}{\left\langle A, \Omega_{\sigma}^{\kappa}(A)\right\rangle_{H S}} \\
& =\epsilon^{2}\left(\frac{1-s^{2}}{1-s^{2} \epsilon^{2}}+\frac{c_{s \epsilon}-\frac{1-s^{2}}{1-s^{2} \epsilon^{2}} c_{s}}{\frac{4}{1-s^{2}} \frac{a_{z}^{2}}{a_{x}^{2}+a_{y}^{2}}+c_{s}}\right)
\end{aligned}
$$

If $c_{s \epsilon}-\frac{1-s^{2}}{1-s^{2} \epsilon^{2}} c_{s} \geq 0$, then

$$
\eta_{\chi_{\kappa}^{2}}(\mathcal{E}, \sigma)=\epsilon^{2} \frac{c_{s \epsilon}}{c_{s}}=\epsilon^{2} \frac{\kappa\left(\frac{1+s \epsilon}{1-s \epsilon}\right) /(1-s \epsilon)}{\kappa\left(\frac{1+s}{1-s}\right) /(1-s)} .
$$

For fixed $s$ and $\epsilon, \eta_{\chi_{\kappa}^{2}}(\mathcal{E}, \sigma)$ might be largely affected by $\kappa$ as well.

\section{Conclusion and outlook}

In this paper, we provide a partial solution to the problem of the tensorization of SDPIs for quantum channels in Theorem 1. In addition, we extend the connection between the SDPI constant for classical $\chi^{2}$ divergence and the maximal correlation to the quantum region in Theorem 18. For a particular QC channel $\mathcal{E}$ and a special quantum state $\sigma$, we observe an extreme scenario, in which the SDPI constant $\eta_{\chi_{\kappa}^{2}}(\mathcal{E}, \sigma)$ ranges approximately from 0 to 1 for different $\kappa \in \mathcal{K}$. This implies that choosing different $\kappa$ might largely affect the rate of contraction of quantum channels. Our numerical experiments (not presented in the paper) conducted for both qubit (i.e., $n=2$ ) and qudit (with $n=3$ ) systems show that the tensorization property (4) seems to hold for any quantum channel $\mathcal{E}$, any reference state $\sigma \in \mathfrak{D}_{n}^{+}$and at least a few weight functions $\kappa$ being tested (e.g., $\kappa_{\min } \equiv \frac{2}{1+x}, \kappa_{\max } \equiv \frac{1+x}{2 x}$ and the family $\kappa_{\alpha}$ with $\alpha=\frac{1}{4}$ and $\alpha=\frac{3}{4}$ ). Proving such tensorization properties is an interesting future work.

Finally, let us comment on the potential generalization of our approach, as well as the limitation. As one might observe, provided that one could show (34), the tensorization of SDPIs is an immediate consequence. However, it seems to be challenging to characterize the class of quantum channels that satisfy (34) in general and this is the reason why we restrict to QC channels and the case $\kappa \geq \kappa_{1 / 2}$ in Theorem 1 . In terms of the validity of (34), we notice that when $\kappa \leq \kappa_{1 / 2}$ (e.g., $\left.\kappa_{\text {min }}\right),(34)$ does not hold even for QC channels. As mentioned above, numerical experiments seem to suggest that the tensorization also holds for $\kappa_{\min }$. Therefore, further understanding of the properties of quantum $\chi_{\kappa}^{2}$ divergences is needed to extend our results.

\section{Acknowledgment}

This work is supported in part by the US National Science Foundation via grants DMS-1454939 and CCF-1910571 and by the US Department of Energy via grant DE-SC0019449. We thank Iman Marvian and Henry Pfister for helpful discussions. Iman Marvian pointed out the possible generalization of Theorem 18 from the canonical purification to any general purification. Henry Pfister introduced us to the topic of the strong data processing inequality for classical noisy channels. We also thank anonymous referees for helpful suggestions.

\section{References}

[1] Venkat Anantharam, Amin Gohari, Sudeep Kamath, and Chandra Nair. On maximal correlation, hypercontractivity, and the data processing inequality studied by Erkip and Cover, Apr 2013. arXiv:1304.6133.

[2] Salman Beigi. A new quantum data processing inequality. J. Math. Phys., 54(8):082202, 2013. DOI: $10.1063 / 1.4818985$. 
[3] Salman Beigi, Nilanjana Datta, and Cambyse Rouzé. Quantum reverse hypercontractivity: its tensorization and application to strong converses, Apr 2018. arXiv:1804.10100.

[4] Hong-Yi Chen, György Pál Gehér, Chih-Neng Liu, Lajos Molnár, Dániel Virosztek, and NgaiChing Wong. Maps on positive definite operators preserving the quantum $\chi_{\alpha}^{2}$-divergence. Lett. Math. Phys., 107(12):2267-2290, 2017. DOI: 10.1007/s11005-017-0989-0.

[5] Man-Duen Choi, Mary Beth Ruskai, and Eugene Seneta. Equivalence of certain entropy contraction coefficients. Linear Algebra Appl., 208-209:29-36, 1994. DOI: 10.1016/00243795(94)90428-6.

[6] Joel E. Cohen, Yoh Iwasa, Gh. Rautu, Mary Beth Ruskai, Eugene Seneta, and Gh. Zbaganu. Relative entropy under mappings by stochastic matrices. Linear Algebra Appl., 179:211-235, 1993. DOI: 10.1016/0024-3795(93)90331-H.

[7] Toby Cubitt, Michael Kastoryano, Ashley Montanaro, and Kristan Temme. Quantum reverse hypercontractivity. J. Math. Phys., 56(10):102204, 2015. DOI: 10.1063/1.4933219.

[8] Fumio Hiai and Mary Beth Ruskai. Contraction coefficients for noisy quantum channels. $J$. Math. Phys., 57(1):015211, 2016. DOI: 10.1063/1.4936215.

[9] Fumio Hiai, Hideki Kosaki, Dénes Petz, and Mary Beth Ruskai. Families of completely positive maps associated with monotone metrics. Linear Algebra Appl., 439(7):1749-1791, October 2013. DOI: 10.1016/j.laa.2013.05.012.

[10] Michael J. Kastoryano and Kristan Temme. Quantum logarithmic Sobolev inequalities and rapid mixing. J. Math. Phys., 54(5):052202, 2013. DOI: 10.1063/1.4804995.

[11] Christopher King. Hypercontractivity for semigroups of unital qubit channels. Commun. Math. Phys., 328(1):285-301, May 2014. DOI: 10.1007/s00220-014-1982-4.

[12] Andrew Lesniewski and Mary Beth Ruskai. Monotone Riemannian metrics and relative entropy on noncommutative probability spaces. J. Math. Phys., 40(11):5702-5724, 1999. DOI: $10.1063 / 1.533053$.

[13] Martin Müller-Lennert, Frédéric Dupuis, Oleg Szehr, Serge Fehr, and Marco Tomamichel. On quantum Rényi entropies: A new generalization and some properties. J. Math. Phys., 54(12): 122203, December 2013. DOI: 10.1063/1.4838856.

[14] Dénes Petz. Sufficiency of channels over von Neumann algebras. Q. J. Math., 39(1):97-108, 1988. DOI: 10.1093/qmath/39.1.97.

[15] Dénes Petz and Mary Beth Ruskai. Contraction of generalized relative entropy under stochastic mappings on matrices. Infin. Dimens. Anal. Quantum. Probab. Relat. Top., 01(1):83-89, 1998. DOI: $10.1142 / \mathrm{S} 0219025798000077$.

[16] Yury Polyanskiy and Yihong Wu. Dissipation of information in channels with input constraints. IEEE T. Inform. Theory, 62(1):35-55, 2016. DOI: 10.1109/TIT.2015.2482978.

[17] Yury Polyanskiy and Yihong Wu. Strong data-processing inequalities for channels and Bayesian networks. In Eric Carlen, Mokshay Madiman, and Elisabeth M. Werner, editors, Convexity and Concentration, The IMA Volumes in Mathematics and its Applications, pages 211-249. Springer New York, 2017. DOI: 10.1007/978-1-4939-7005-6_7.

[18] Maxim Raginsky. Strong data processing inequalities and $\Phi$-Sobolev inequalities for discrete channels. IEEE T. Inform. Theory, 62(6):3355-3389, June 2016. DOI: 10.1109/TIT.2016.2549542.

[19] Cambyse Rouzé and Nilanjana Datta. Concentration of quantum states from quantum functional and transportation cost inequalities. J. Math. Phys., 60(1):012202, 2019. DOI: $10.1063 / 1.5023210$.

[20] Mary Beth Ruskai. Beyond strong subadditivity? Improved bounds on the contraction of generalized relative entropy. Rev. Math. Phys., 06(5):1147-1161, 1994. DOI: 10.1142/S0129055X94000407.

[21] K. Temme, M. J. Kastoryano, M. B. Ruskai, M. M. Wolf, and F. Verstraete. The $\chi^{2}$-divergence and mixing times of quantum Markov processes. J. Math. Phys., 51(12):122201, 2010. DOI: $10.1063 / 1.3511335$.

[22] Ramon van Handel. Probability in high dimension, 2016. available at https://web.math. princeton. edu/ rvan/APC550.pdf.

[23] Mark M. Wilde. Quantum Information Theory. Cambridge University Press, 2013. DOI: 10.1017/CBO9781139525343. 
[24] Aolin Xu and Maxim Raginsky. Converses for distributed estimation via strong data processing inequalities. In 2015 ISIT, pages 2376-2380, 2015. DOI: 10.1109/ISIT.2015.7282881. 